\title{
MAGNETOSPHERIC STRUCTURE OF ROTATION-POWERED NEUTRON STARS
}

\author{
JonATHAN ARONS \\ Astronomy Department and Physics Department, University of California at Berkeley \\ Institute of Geophysics and Planetary Physics, Lawrence Livermore National Laboratory
}

\begin{abstract}
I survey recent theoretical work on the structure of the magnetospheres of rotation-powered pulsars, within the observational constraints set by their observed spindown, their ability to power synchrotron nebulae and their ability to produce beamed collective radio emission, while putting only a small fraction of their energy into incoherent $\mathrm{X}$ - and gamma radiation. I find no single theory has yet given a consistent description of the magnetosphere, but I conclude that models based on a dense outflow of pairs from the polar caps, permeated by a lower density flow of heavy ions, are the most promising avenue for future research.
\end{abstract}

\section{What do we know?}

'Is there any point to which you wish to draw my attention?'

'To the curious incident of the dog in the night-time.'

'The dog did nothing in the night time.'

'That was the curious incident', remarked Sherlock Holmes.

Silver Blaze, by A. Conan Doyle

Why do not radio pulsars appear as noisy barking dogs in the galactic night? This question is the central one which needs to be addressed by all theories of the pulsar magnetosphere. After all, the Crab pulsar broadcasts a total luminosity in rotational energy to the surrounding cosmos equivalent to $10^{3}$ large nuclear wars being fought per second on each square meter of its surface, yet only a few tenths of a percent of this energy appears as radiation coming from the immediate environs of the star, and less than one part in $10^{6}$ appears in pulsed radio emission. Common pulsars are less spectacular-only one nuclear battle per square meter per second, with no visible sign of the object except the much smaller trickle of energy coming out in the coherent radio emission. Thus, we have to model the collective electromagnetic emission from a natural system, and also understand how such an effective muzzle has been put on the processes which could dissipate the total energy lost into incoherent emission, all with little help from higher frequency observations in determining the magnetospheric configuration of plasma and fieids which give rise to the collective emissions.

Such a task is very attractive to people who like the pursuit of pure, bottoms up theory building, with the orientation heavily weighted toward explaining the most complex and least energetic phenomena, the coherent radio emission which is the focus of this conference. After all, these are the data we have, with rich detail. However, the constraints that come from the few successful high frequency detections of rotation-powered pulsars, and from the larger number of upper limits in the $\gamma$ - and $\mathrm{X}$-ray bands, are at least as important as the magnetospheric issues that touch on the radio emission directly. Therefore, before turning to the current state of magnetospheric theory, I will summarize a number of constraints that any proposed model should meet, in order of their energetic significance.

To be an honest propagandist, I need to preface my remarks with a disclaimer. I have not worked on issues of magnetospheric structure since I studied some aspects of pair creation and the nature of the inner magnetospheric flows which might give rise to the observed emission, more than 5 years ago. Nevertheless, I accepted the organizers' invitation to give this talk, as it gave me a good excuse to catch up with what my colleagues have been doing. Therefore, I can afford to take an Olympian view of the field, since I do not have a particular magnetospheric model to push on you (although I will exercise the usual prerogative of reviewers and draw attention to some of my own work where I think it is relevant). I do have strong opinions, which color what I emphasize. I also will not be able to give equal time and attention to all the efforts made in this area-for those authors who feel slighted or forgotten, my apologies in advance.

\section{Spin down}

Because pulsar radio emission taps such a tiny fraction of the energy lost from an isolated, magnetized, rotating neutron star, studying pulsars through the physical properties of their radio emission is rather like studying the sun solely through its coherent radio emission - if all we could see of our own star was 
the tiny trickle of energy coming through the complex phenomena of the type II and III solar bursts, we would not have much of a clue as to the nature of the underlying beast. Fortunately, the wonderfully stable underlying clock seen in radio pulsars reveals the essential truth-only rotating magnetized neutron stars can provide the stability seen in mean pulse profiles. The arguments for this conclusion are as old as our knowledge of pulsars, and are fully summarized by Manchester and Taylor (1977), for example. The stability of the clock, operationally constructed by averaging many individual pulses together (usually more than $10^{2}$ ), allows us to determine not only the period $P$, but also its derivative $\dot{P}$ and sometimes even $\ddot{P}$.

Observations reveal a rate of rotational energy loss $\dot{E}_{\mathrm{R}}=4 \pi^{2} I \dot{P} / P^{3} \approx 4 \times 10^{31} I_{45} \dot{P}_{15} / P^{3} \mathrm{erg} / \mathrm{s}$, where $I_{45}$ is the moment of inertia in units of $10^{45} \mathrm{cgs}, P$ is measured in seconds and $\dot{P}_{15} \equiv$ $\dot{P} / 10^{-15}$. With scattered exceptions, this energy just disappears from the view afforded by detectors cperating above the radio band, while only a tiny fraction (between $10^{-6}$ and $10^{-2}$ ) showre $" p$ in the collective radio emission.

\section{Electromagnetic spindown}

Originally, this energy loss was ascribed to the emission of large amplitude electromagnetic waves with vacuum propagation characteristics (Ostriker and Gunn 1969). At the same time, the energy loss was ascribed to the electromagnetic torques associated with a force-free magnetospheric current system running all the way from the star to the distant nebula or interstellar medium (Goldreich and Julian 1969), and to the torques associated with an inertially loaded, relativistic magnetohydrodynamic wind (Michel 1969). All of these ideas are really manifestations of the same thought: magnetospheric energy flow is dominated by a large scale Poynting flux of electromagnetic energy. This basic idea offers a proposed solution to the silence of the barking dog-at most, the electromagnetic energy oscillates at the rotation frequency, far below that of any directly observable photons.

\section{Dominance of Poynting flux}

In order to see why all these ideas really are variations on the same theme, I construct the widely used "vacuum" formula for spindown by an order of magnitude argument. The electric field is generated by rotation of the magnetic field, so in order of magnitude its value is $E \sim(\Omega r / c) B$, where $\Omega=2 \pi / P$. Assume there is a component of the electric field of this order of magnitude lying in planes of rotational latitude. Suppose the electromagnetic energy den- sity is large compared to the plasma energy density out to radii $r=R_{\mathrm{L}}=c / \Omega=5 \times 10^{4} \mathrm{~km}$. Finally suppose that at this "light cylinder" radius, some mechanism causes the formation of a swept back toroidal magnetic field $B_{\phi}\left(R_{\mathrm{L}}\right) \approx B_{\mathrm{p}}\left(R_{\mathrm{L}}\right)$, where $B_{\mathrm{p}}$ is the poloidal magnetic field. Then there is a radial component of the Poynting flux across the sphere of radius $R_{\mathrm{L}}$ corresponding to the total energy loss rate

$$
\dot{E}_{\mathrm{R}} \sim 4 \pi R_{\mathrm{L}}^{2} \frac{c}{4 \pi}\left[(E \times B) \cdot \hat{e}_{\mathrm{R}}\right]_{r=R_{\mathrm{L}}} \cong-\Omega R_{\mathrm{L}}^{3} B^{2}\left(R_{\mathrm{L}}\right) .
$$

If the magnetic field at radii $R_{*} \ll r \leq R_{\mathrm{L}}$ is dipolar, with magnitude $\mu / r^{3}$, eq.(1) yields

$$
\dot{E}_{\mathrm{R}} \approx-\frac{\mu^{2} \Omega^{4}}{c^{3}} f(\chi)
$$

where $f(\chi)$ is some geometric factor depending on the angle $\chi$ between the rotation and the dipole axes and possibly on other physical factors. The full calculation of the vacuum fields (Deutsch 1955) tells us that $f_{\text {vac }}(\chi)=2 \sin ^{2} \chi / 3$. The force-free current idea and the MHD wind model do not specify $f(\chi)$, since as originally presented they do not provide a complete description of the field structure in their estimates of the torque. Equating eq.(2) to the rate of loss of energy from the star gives the usual proportionality of $\dot{\Omega}$ to $\Omega^{3}$ and allows us to find the magnetic moment to be $\mu=10^{30}\left(P \dot{P}_{15}\right)^{1 / 2} \mathrm{cgs}$ if we assume $f(\chi) \sim 1$, a result essential to all the elaborate modeling of pulsar evolution.

Eq.(2) requires the predominance of electromagnetic forces within the magnetosphere so that the energy losses are mostly carried by the Poynting flux, a condition clearly satisfied by the vacuum model and also satisfied by assumption in the Goldreich and Julian suggestion and in Michel's original form of the MHD wind model. In addition, there is an implicit assumption, that the field pattern is steady in the corotating frame. The other required feature is a toroidal magnetic field comparable to the poloidal component, at distances comparable to $R_{\mathrm{L}}$. The displacement current is the origin of this $B_{\phi}$ in the vacuum model, while in the Goldreich and Julian idea, $B_{\phi}$ has its origin in an electric current flowing from the polar regions of the star with density $J_{\|}=B / P$, a current which is just right to support $B_{\phi} / B_{\mathrm{p}} \sim-1$ at $r \sim R_{\mathrm{L}}$. In Michel's original form of the MHD model, the origin of $B_{\phi}$ is not addressed in terms of the behavior of the inner magnetosphere, since he, in common with rnany subsequent authors (Kennel, Fugimura, and Okamoto 1983) adopted the split monopole model of the poloidal field, in which there are no closed regions at all in the magnetosphere. Instead, his wind is driven by the global electromagnetic stresses 
alone, which guarantees the light cylinder as the location of the region where $B_{\phi} / B_{\mathrm{p}} \sim-1$.

\section{Examples of $n<3$ : Plasma or evolution?}

As a result, studies of spindown alone have a hard time telling us much of the physics in the magnetosphere: the spindown rate depends only on a very low moment of the magnetospheric structure. Nevertheless, this electromagnetic idea is subject to some degree of testing from timing data alone, since as stated it predicts the "braking index" $n=\Omega \ddot{\Omega} / \dot{\Omega}^{2}$ will be exactly equal to 3 , if evolution affects neither the field strength nor the obliquity [or other parameters influencing eq.(2)]. Testing this idea requires timing short period pulsars with large values of $\dot{P}$ so that rotation phase accumulates quickly. In these, the spin down is not entirely steady, but shows the restless wandering most often modeled as "white noise in the torque" (really, noise in the ratio of the torque to the moment of inertia). Such phenomenological models have been applied to the timing data of the Crab and Vela pulsars, to PSR 1509-58 and to the "Crab clone" PSR 0540-69 in the Large Magellanic Cloud, with the result that in all cases $n<3$ (Groth 1975, Lyne, Pritchard, and Smith 1988, Cordes and Downs 1985, Manchester, Durdin, and Newton 1985, Manchester and Peterson 1989)! The most extreme case is the LMC pulsar, with $n=2$.

Evolutionary effects are one possible interpretation. If we assume $\mu(t)$ and $\chi(t)$ are the only parameters affecting the torque, and these are not strict constants, then the observed braking index would be

$$
n=3-\frac{\Omega}{|\dot{\Omega}|}\left(\frac{\dot{\mu}}{\mu}+\frac{\partial \ln f}{\partial \chi} \dot{\chi}\right) .
$$

In the vacuum model, either increase of the magnetic moment or $\chi \rightarrow \pi / 2$, on a time scale comparable to the spin-down time of these relatively young objects, would explain the observations (see Blandford and Romani 1988). Increasing magnetic moments would tell us something about the evolution of the interior (crust or core-crust boundary, most likely), while alteration of $\chi$ would tell us something about the interplay between magnetospheric torques and the structure of the crust (Michel and Goldwire 1970, Goldreich and Julian 1970). No progress can be made on the possibilities of varying $\chi$, however, until some decision can be made about how the torques do depend on obliquity. Further on, I describe more modern theories of the magnetosphere which have quite different predictions for $f(\chi)$ than the vacuum model. It should be said, however, that explaining small braking indices in these relatively young, short period pulsars by having $\chi$ approach either 0 or $\pi / 2$ on the spin down time scale would leave these objects either as almost aligned or almost orthogonal rotators when they reach old age. Since most pulsars are not in either of these geometric states (Lyne and Manchester 1988), variations in $\chi$ can be used to explain small braking indices only if one simultaneously appeals to almost all pulsars being born with rather long periods (Emmering and Chevalier 1989 and references therein) and reaching death as radio emitters before they experience much spin down and become aligned or orthogonal.

I do not think this combination of hypotheses is very likely for the following reason. There are several dozen radio pulsars such as PSR $1951+32$ in CTB 80 with ages $\sim 10^{5}$ years which will live 10 100 spindown times until they die as radio objects. If they align or counter-align on the spindown time, one would expect at least as many pulsars near the death line which have the broad pulses possibly characterizing almost aligned rotators, or the frequency independent pulse-interpulse structure possibly characterizing radiation from opposite poles. Since the number of objects with such "unusual" pulse profiles is small compared to the number of objects with standard beaming morphologies, alignment or counteralignment on the spindown time scale probably does not occur. Therefore, if evolution is the origin of $n<3$, some form of increase of the dipole moment associated with the field lines which reach the outer magnetosphere is likely to be the origin of the effect in these "young" objects.

From the point of view of magnetospheric theory, having a characteristic radius for full development of the torque differing from $R_{\mathrm{L}}$ is a more entertaining possibility. Suppose, just for the sake of argument, that $B_{\phi} / B_{\mathrm{p}} \sim-1$ is achieved at some radius $R_{\mathrm{A}}=R_{\mathrm{L}}\left(\Omega R_{*} / c\right)^{p}=R_{*}^{p} R_{\mathrm{L}}^{1-p}$. Carrying through the estimate of the rotational energy loss yields eq.(1) with $R_{\mathrm{A}}$ replacing $R_{\mathrm{L}}$, and an energy loss rate $\propto \Omega^{4-3 p} \mu^{2}$. One can match the observed braking index of the Crab (2.5) with $p=1 / 6$, and that of PSR 0540-69 with $p=1 / 3$, for example, corresponding to $R_{\mathrm{A}} / R_{\mathrm{L}}=0.43,0.16$ in these two cases. If the magnetic field were really a monopole, we would have $p=1$.

The only way to move $R_{\mathrm{A}}$ noticeably inwards from the light cylinder is if there is a substantial conduction current flowing on polar field lines. For the moment I will simply define these as the field lines of the undisturbed dipole which would have closed at radii larger than $R_{\mathrm{A}}$. If the stresses in the magnetosphere are negligible, then these currents must flow at least to $r \sim R_{\mathrm{L}}$, where some sort of stress must intervene to cause the current 
to flow further-see below. In this now classical view, the polar flux tubes then each intercept an area $\sim \pi R_{*}^{3} / R_{\mathrm{L}}$ on the stellar surface. If $R_{\mathrm{A}} \ll R_{\mathrm{L}}$, either the conduction current density in the polar flux tube greatly exceeds the Goldreich and Julian value of $B / P$, or the area intercepted on the surface has to be much bigger. I used to greatly like this idea. However, moving the Alfvén radius in from the light cylinder by factors of 2 to 10 is quite extreme, since it requires a huge stress to be exerted on the inner magnetosphere, all without producing exorbitant amounts of observable radiation. As a result, I am mildly in favor of any magnetic field variation which can manifest itself as an increase of the dipole moment as perceived by a test coil placed in the outer magnetosphere as the origin of the small braking indices, although I think we are a long way from being able to model them in a credible way.

\section{Spin up line and dipole fields of millisecond pulsars}

There are other ways in which timing can shed light on questions of magnetospheric structure, so long as one asks only about global properties. The discovery of the millisecond pulsars revealed to us systems with $R_{\mathrm{L}}$ only a few times the stellar radius. Since timing measures torques, which depend on the field at $R_{\mathrm{L}}$, in standard pulsars one has no direct handle on the surface field, which could be much more complex (and indeed, almost, certainly is a lot messier, if the field grows in the surface layers of the crust). In the millisecond pulsars, one might suspect the weak fields entering into the torque might have strong multipoles contributing.

The millisecond pulsars are thought by many to be the evolutionary descendents of standard radio pulsars, as the result of magnetic field decay and spin up by accretion in a binary during the time when the fields reach small values. Let me assume all neutron stars have such an evolutionary history. At the same time, recent observations of $\gamma$-ray bursts have shown the presence of line features in the $\mathrm{X}$-ray spectrum characteristic of cyclotron scattering in a field whose surface strength is $\sim 10^{12}$ Gauss. If $\gamma$-ray burst sources are neutron stars, as they certainly seem to be as judged by these spectral line data, they must be drawn from the population of galactic neutron stars at large, whose age is comparable to that of the galaxy, and not from the smaller subset with non-decayed fields ${ }^{1}$. One might try to reconcile the weak dipoles

\footnotetext{
${ }^{1}$ One can get around this to some extent by shortening the recurrence time for bursts in one object to be less than 30 years, yet longer than 10 years to avoid the observed lack of recurring flashes in most bursters (van Paradijs 1989). While
}

inferred from the torques on the millisecond pulsars with the strong surface fields inferred from a couple of $\gamma$-ray bursters by supposing the magnetospheric fields of old objects are really dominated by higher order multipoles. If $B \propto r^{-(l+1)}$, one can reconcile the strong surface fields of at least some $\gamma$-ray burst sources with the apparently weak fields of the millisecond pulsars if $l \geq 5$, while the true dipole field is at least a factor of 100 weaker at the stellar surface. Such a model predicts a braking index well in excess of 3 , but it will be a long time before these small $\dot{P}$ objects have their braking indices measured.

However, a different aspect of timing information can be used to require the field to be dipolar, even in the old objects. One can readily show (Arons 1990, to be submitted to Nature) that the limiting spin rate to which a neutron star can be accelerated during accretion yields a spin up line in the $P, \dot{P}$ diagram of $\dot{P} \propto P^{(8-2 l) / 3}$ for $l \geq 2$. For $l=2$ (dipole), this line does form an envelope for most of the millisecond pulsars, as is well known, while for $l>2$ (quadrupole and more complicated), the spin up line is in total disagreement with the data. Therefore, one cannot reconcile the gamma ray burst sources and the millisecond pulsars with a single evolutionary history. If field decay and growth does play a role, then it must be different for objects which experience strong accretion in binaries (the millisecond pulsars) and those which do not (the common field objects, from which most $\gamma$ ray burst sources are drawn), a conclusion drawn by others on different grounds (Shibazaki et al. 1989). In addition, I can draw the conclusion that the internal currents are relatively deeply buried, since usually the dipole component is dominant only well outside the interior regions where the currents flow.

I also remark that arguments of this kind presuppose the dependence of the torque on $\chi$ and any other parameters to be something like that of the vacuum rotator. Almost everyone believes this to be true, although in fact this belief has no objective basis. I will discuss shortly a theory with quite different implications.

\section{Excitation of plerions-pulsars emit heavy ions!}

Despite the interesting information which can be gleaned about the global characteristics of the field from timing studies, a real understanding of the physics will occur only if we can observe photon emission from something which probes the physics of the magnetosphere more directly - without such probes, we are in the position of the searches for

not yet formally excluded, the chances are not large that this loophole is actually open. 
dark matter, always creating indirect constraints without having a direct view. One possibility is to model the radio emission itself, but since this channel contains such a negligible fraction of the total energy, it is important to look first at other forms of emission whose modeling is less complex. After the spindown energy loss itself, emission from the surrounding medium is the next in energetic importance, since in some cases a substantial fraction of the spin-down energy reappears in the incoherent synchrotron emission from the surrounding nebula (a "plerion"). My attitude here is to ask, can we identify a successful model of the acceleration mechanism in a plerion? If the answer tells us something of the composition of what comes out of the pulsar, then the results form exterior boundary conditions on any model of a pulsar magnetosphere. In the interests of brevity, I will restrict discussion to the Crab Nebula and its pulsar-most of the inferences should apply to all plerions, but the data on other systems are not nearly as complete, causing the resulting discussion to become even more model dependent.

Obviously, the Crab pulsar sends energy outwards in some low entropy form, to be delivered to the nebular plasma with sufficient energy/particle to create a relativistic plasma in the nebula. This plasma has much more entropy than whatever carries the energy from the pulsar to the nebula-while nonthermal, the spectrum of radiating particles has a broad distribution in energy space, roughly modeled as several joined power laws. The nebular optical, $\mathrm{X}$ - and gamma-ray emission is a more or less immediate probe of the pulsar's instantaneous output, since the synchrotron losses age the radiating particles in a time short compared to the nebular and pulsar lifetimes. As judged by the direction of the optical polarization in the inner nebula (Scargle 1969), the rotation axis of the pulsar is along the long axis of the nebula, with the $\mathrm{X}$ - and gammaray source forming a fat torus lying in the region of the pulsar's rotational equator (Aschenbach and Brinkman 1975, Pelling et al. 1987).

\section{Wave models}

The vacuum theory easily satisfies the low entropy condition for the carrier--a $30 \mathrm{~Hz}$ wave has all the energy in 4 field degrees of freedom, and is perfectly invisible. However, there has never been a successful theory of how this wave can convert its energy into an electron (or possibly positron) distribution spreading over $\sim 6$ decades in energy space. The wave can propagate into the nebular plasma only if $\omega_{\mathrm{p}}^{2} \ll \Omega^{2}$, where the relevant relativistic plasma frequency is $\omega_{\mathrm{p}}^{2}=4 \pi e^{2} N / m \gamma$. The basic energy/particle is that of the oscillatory momen- tum $p_{\text {osc }} \cong e B_{\text {wave }} / \Omega \sim 10^{3} \mathrm{MeV} / c$ given to each particle by the wave. Then $\gamma \sim 1+e B_{\text {wave }} / m c \Omega$, and propagation requires

$$
N<\frac{\Omega B_{\text {wave }}}{2 \pi c e}=\frac{\eta_{\mathrm{R}}}{e} \approx 10^{-4} \frac{B_{\text {wave }}}{10^{-4} \mathrm{G}} \frac{33 \mathrm{~ms}}{P} \mathrm{~cm}^{-3} .
$$

The charge density $\eta_{R}$ reappears in the theory of the magnetosphere itself as the density of elementary charges neuded to support the electric field associated with the corotation of charged particles with the star. Here, it corresponds to the cutoff density of an electromagnetic wave whose frequency is the rotation frequency of the pulsar and which has amplitude large enough to drive particles relativistic.

Synchrotron models of the nebular radiation suggest the density of radiating particles in the $\mathrm{X}$ - and gamma-ray source is $\sim 10^{-5} \mathrm{~cm}^{-3}$, so the wave can propagate. However, it is violently unstable (Max 1973, Arons, Norman, and Max 1977), and it radiates its energy as incoherent $\gamma$-rays in a few cycles, for densities in excess of a few percent of $\eta_{\mathrm{R}} / e$ (Asséo, Kennel, and Pellat 1978). All of this dissipation is a consequence of the wave having $E_{\text {wave }}>B_{\text {wave }}$ in the medium. When this inequality is satisfied, the dominant physics is the relativistically large work being done on the particles, as is made obvious by transforming to the frame where $B_{\text {wave }}=0$.

One might think this rapid dissipation is a good thing, since it forces the wave to dump all of its energy in a thin layer, formed where the wave momentum flux $\dot{E}_{\mathrm{R}} / 4 \pi r^{2} c$ approximately equals the nebular pressure $p_{\mathrm{p}} \sim 10^{-8}$ dyne $\mathrm{cm}^{-2}$, a distance $R_{b}=\left(\dot{E}_{\mathrm{R}} / 4 \pi p_{\mathrm{p}} c\right)^{1 / 2} \sim 0.1$ light years from the pulsar. Thus, one could explain the well-known "cavity" in the nebular emission (Schmidt, Angel, and Beaver 1979) by using the low frequency wave to blow this pressure-confined bubble, with the accelerated particles coming from the nebular plasma, whose origin would be evaporation from the emission line filaments. The "wisps" in the optical synchrotron emission (Scargle 1969), which appear at. about this radius, might be attributed to the wave dissipation at this "working surface". However, the model spends too much of its energy in $\gamma$-ray emission right in the boundary layer between wave and nebula, and puts too little into particle heating. In addition, I am unaware of any means of converting the particle heating into the broad, power-lawlike distributions needed to explain the synchrotron source-both theories of the wave-particle interaction and the one plasma simulation done of the nonlinear state of the breakdown of a strong wave in a plasma (Leboeuf $e t$ al. 1982) show spectra more like a delta function at a characteristic energy, rather than the power-law-like distribution which would 
have to diffuse away from the interface in order to explain the nebular spectrum. The same arguments suggest that if there is a particle density even as small as a few tenths of a per cent of $\eta_{R} / e$ near the pulsar's light cylinder, the wave would never survive in low entropy form to distances more than a few times $R_{\mathrm{L}}$. Of course, there are thinkable models with no particles at all in the outer magnetosphere (see below), so this objection may not be fatal. However, the inability of the wave models to drive a synchrotron source in the surrounding medium with characteristics anything like the observed nebula suggests that wave heating models of plerionic excitation are not the correct representation of reality. In addition, the nebular magnetic field cannot be the wound off field from the pulsar, since the wave cannot penetrate the plasma, requiring a whole separate theory for the magnetism of plerions.

\section{Wind models}

A relativistic MHD wind is the physical opposite of the "racuum" wave model. Relativistic speeds are needed, since we are trying to feed an exterior medium in which at least $10 \%$ of $\dot{E}_{\mathrm{R}}$ reappears as highly relativistic particles. The pulsar's magnetosphere must emit sufficient plasma to short out all electric fields in the proper frame of the flow. The electric field in any other frame then always satisfies $E<B$. The lack of electrification in the proper frame suggests there will be little radiative dissipation in the flow, thus satisfying the lack of a barking dog near the pulsar. This flux freezing condition requires densities in the outflow to be large compared to $\eta_{\mathrm{R}} / e$, corresponding to a particle loss rate from the pulsar large in comparison to $\dot{N}_{\mathrm{R}}=2 \pi R_{*}^{3} \Omega \eta_{\mathrm{R}}\left(R_{*}\right) / q \sim$ $2 \times 10^{34}(33 \mathrm{~ms} / P)^{3 / 2}\left(\dot{P} / 4 \times 10^{-13}\right)^{1 / 2} Z^{-1}$, where $I$ have assumed the magnetic moment can be estimated from eq.(2) and the mean charge of the particles in the flow is $Z$.

In contrast to the wave, which cannot penetrate the nebular plasma, the wind idea is an efficient explanation of both the cavity in the emission around the pulsar, and of the nebular magnetic field. The cavity is understandable if the outflow is highly supersonic (with respect to the magnetosonic speed), with little radiation in the proper frame until the flow halts in a shock at the momentum balance surface $R_{b}$,[Rees and Gunn (1974), who mixed together vacuum wave and MHD wind concepts in a way not consistent with the fundamental physics, but who were the first clearly to identify shock excitation as a possible origin of the nebular particle acceleration]. The nebular magnetic field can then be explained as the advection of the toroidal field lines in the post-shock flow which fills most of the nebular volume, a realization of Piddington's (1957) early suggestion for the origin of the nebular magnetism. The sense of the linear polarization in the optical nebula (parallel to the pebula's long axis) is consistent with this idea. The net circular polarization of the whole nebula in the radio (Weiler 1975 ) is not consistent with a toroidal field. However, these observations are only of marginal significance, and in any case they refer to the magnetic structure in the total nebular volume, where the flow of the interfilamentary plasma over the "rocks" in the stream formed by the emission line filaments probably cause the field to be drawn out in linear, "cometary" structures downstream of the filaments (Chevalier 1984, Fesen 1990, Michel et al. 1991).

\section{Plasma dominated wind excitation}

Kennel and Coroniti (1984a, b) have carried out the most elaborate implementation of the MHD wind idea; Kundt and Krotscheck (1980) earlier published related ideas. Because the nebula expands slowly $\left(\dot{R}_{\text {neb }} \sim 1000 \mathrm{~km} \mathrm{~s}^{-1}\right)$ and the sound speed of the shocked plasma from the pulsar is $c_{s} \sim c / \sqrt{3} \gg \dot{R}_{\text {neb }}$ (they assume an isotropic postshock plasma), the shock must convert most of the flow energy into "thermal" energy downstream-if the downstream energy flux is still predominantly electromagnetic, the flow speed is $\sim c$ throughout the post-shock region and the shocked bubble cannot fit into the cavity confined by the inertia of the surrounding interstellar (or, more likely, circumstellar) matter. The ideal MHD jump conditions show that non-relativistic downstream flow requires the upstream flow to be plasma dominated also-the ratio of the upstream Poynting flux to the total plasma energy flux (including rest mass energy)

$$
\sigma \equiv \frac{B_{1}^{2}}{4 \pi m N_{1} \gamma_{1} c^{2}}
$$

must be much less than unity. Fitting the post shock flow into the cavity requires $\sigma \approx 0.003$, if the nebular expansion is ignored (this is $\mathrm{OK}$, to zeroth order in $\left.\dot{R}_{\text {neb }} / c_{s}\right)$. Including the time-dependent effect of nebular expansion requires $\sigma<0.006(\mathrm{Em}$ mering and Chevalier 1987).

Thus, at the shock, the magnetic field broadcast by the pulsar is more than an order of magnitude weaker than what one would expect from a Poynting flux dominated wind, contrary to the most elementary spin-down ideas outlined above. One way to make this surprising conclusion consistent with the expectation of electromagnetic dominance at the source is to observe that the magnetic field of the wind in the vicinity of the pulsar's magnetic equator is composed of field lines 
wound up from opposite poles of the dipole - the magnetic field carried out forms "stripes" of oppositely directed toroidal $B$, each of width $\sim R_{\mathrm{L}} \sim$ $1000 \mathrm{~km} \ll R_{b} \sim 10^{9} R_{\mathrm{L}}$. Magnetic field reconnection (and annihilation) then might reduce the magnetic energy in the proper frame of the flow, so that one could have $\sigma \gg 1$ in plasma leaving the outer magnetosphere while $\sigma<10^{-2}$ at radii where the wind is shock decelerated. Coroniti (1990) has shown that such annihilation of magnetic energy might occur if the Crab pulsar is sufficiently close to being an orthogonal rotator $\left(\chi \sim 80^{\circ}\right)$, and the plasma is sufficiently dense. The main worry of this idea is the almost complete conversion of the magnetic energy into heat in the proper frame of the wind, which might reappear as radiation. Because of relativistic beaming, this would appear as an intense unpulsed radiation source on top of the pulsar. Coroniti explains the absence of intense unpulsed emission at the angular position of the pulsar as a consequence of adiabatic losses in a pure pair plasma-most of the dissipated energy goes into mildly accelerating the flow. In chapter II, I briefly describe two other ideas deriving from the physics of the magnetosphere which provide alternatives to this wind zone origin of small $\sigma$ in the asymptotic flow.

Furthermore, the Kennel and Coroniti model explains why there is approximate equipartition between between the nebular magnetic field and the synchrotron emitting plasma. Immediately behind the shock, the energy is more than $99 \%$ in thermal pressure and plasma flow energy with speed $c / 3$. But this flow rapidly decelerates, all the while conserving the magnetic flux. This deceleration crowds the field lines together until the magnetic pressure becomes comparable to the total, effectively stiffening the equation of state and preventing further deceleration. Thus, in most of the nebular volume, the expansion speed is that of the outer boundary with equipartition between the energy of magnetic fields and relativistic particles-no magical appeals to "turbulence" are needed, as is consistent with the relatively orderly field needed to explain the high polarization in the inner half of the nebula.

The greatest success of this scheme is the construction of an approximate spectrum of the nebula in crude accord with the observed spectrum, as it would be seen in unresolved observations of the nebula. If the accelerated particles creating the nebular X-rays gain and lose energy just once, roughly $10^{38}$ new particles must be accelerated to energies $\sim 10^{5}-10^{7} m c^{2}$ every second. The shock terminating the wind provides this one pass acceleration, if the number of particles flowing out from the pulsar is at least four orders of magnitude greater than $\dot{N}_{\mathrm{R}}$. Kennel and Coroniti suppose the pulsar provides these particles in the form of an electron-positron pair plasma, as might be expected from some of the magnetospheric models proposed by earlier authors. They assume the relativistic shock wave in the low $\sigma$ flow creates heat in the form of power-law distributions of electrons and positrons. Then they show that the flow downstream from the shock, in which the magnetic field is compressed and the highest energy particles lose energy to synchrotron radiation, leads to a synchrotron emissivity distribution in space whose output can be fit to the nebular Xand gamma-ray spectrum, by proper adjustment of the parameters of the assumed power laws. The parameters of the power law so obtained are consistent with the expected dynamics of the pair wind (bulk flow Lorentz factor $\gamma_{1} \sim 10^{6}$, for example), and supply a value for $\sigma$ in the same range as obtained from the MHD dynamical model. Finally, the fit to the $\mathrm{X}$ - and gamma-rays correctly predicts the near IR and optical spectrum of the nebula, a rare achievement for any model of a high energy astrophysical system. However, their model fails to account for the radio emitting particles in the nebula, and so should be thought of as describing the angular sector around the rotational equator of the pulsar where the torus of high energy photon emission lies. The nebular radio emission then must be attributed to a different population of particles supplied by the pulsar. I think this is quite possible, but further discussion would take me too far afield here.

\section{Shock structure-pairs and ions}

As models of high energy astrophysical systems go, the shocked MHD wind model is stunningly successful. Taken at face value, it suggests that pulsars must emit lots of pairs, since this is the only way to get a particle number output large compared to $\dot{N}_{\mathrm{R}}$. However, it is pure assumption that essentially all the flow energy is turned into power-law downstream distributions. The magnetic field from the pulsar has been wound up in a spiral with $\sim 10^{9}$ turns. Therefore, the shock has a transverse geometry, so one cannot appeal to the ever-popular diffusive first order Fermi process to explain the particle acceleration. Furthermore, much of the energy in the hypothesized power laws is in particles whose Larmor radius is comparable to the expected thickness of such a shock, so the acceleration problem is closely related to that of the structure of transverse relativistic shocks.

In recent work done in collaboration with $M$. Hoshino, Y. Gallant, A. B. Langdon and C. E. Max, I have studied the structure of transverse magnetosonic shocks, using a combination of particle-incell plasma simulations and theory. Some aspects of 
our results are reported in our contributed paper to this conference. We find that the shock structure is dominated by magnetic reflection of the whole plasma from the increasing magnetic field in the shock front. When set into gyrational motion by the reflection, the plasma forms a complete or partial ring in momentum space, which acts as a synchrotron maser. The emission of intense collective radiation at the relativistic cyclotron fundamental and at high harmonics thermalizes the plasma within a few cyclotron periods. The shocks have an intense, rather coherent electromagnetic precursor propagating upstream.

The most important results for our purposes here are that shocks in a pure pair plasma produce perfect Maxwellians, in contradiction to the assumptions of the pure pair form of the wind model! If, however, the upstream plasma is non-symmetric, with an admixture of heavy ions which are- a small population by number density but which contain the largest component of the flow energy, then the downstream positrons are efficiently accelerated into a power-law distribution with properties appropriate for the wind model. The origin of this acceleration is simple. The pairs are first shocked and heated to relativistic Maxwellians, while the heavy ions undergo the first phases of their reflection from the shock front. The heavy ions eventually reflect from the shock front and thermalize through the maser emission of extraordinary modes. Most of the emitted energy is at high harmonics of the relativistic ion-cyclotron frequency (based on upstream parameters). The waves emitted have elliptically polarized electric fields which rotate around the background magnetic field in the same sense as the gyration of an ion and of a positron. When the spectrum of ion waves extends to harmonics $l \sim m_{i} / m_{ \pm} \geq 1836$, the heated positrons efficiently absorb the high harmonic ion waves through their cyclotron resonance. This absorption produces a power-law spectrum of positrons. If absolutely all the energy in the ion waves is absorbed by the positrons as fast as the ions emit it, a simple quasi-linear model suggests the downstream positron spectrum would be very flat, with $N(E) d E \propto E^{-1} d E$, with the positron spectrum extending up to $\left(m_{i} / m_{ \pm}\right) \gamma_{1}>10^{9}$. The simulations suggest the spectrum is steeper, with slope between 2 and 3 , as is required by the macroscopic models. Thus, we are led to suggest that the nebular radiation from plerions comes from shock accelerated positrons. Furthermore, we conclude that the pulsar wind must have its flow energy primarily in heavy ions, although by number it is primarily composed of electrons and positrons. Finally, in order to generate collective ion-synchrotron emission at sufficiently high harmonics, the momentum dispersion of the reflected ions must be very small in the shock frame, with $\Delta \gamma<\left(m_{ \pm} / m_{i}\right) \gamma_{1}$. This requires the upstream flow to be very cold, with velocity dispersion in the upstream proper frame $v_{\text {th }} / c<\sqrt{m_{ \pm} / m_{i}}$. Even with strong adiabatic losses in the outflow, this excludes thermal mechanisms from consideration as the origin of the wind.

I conclude, therefore, that the Crab Nebula, and probably other plerions, points toward pulsars losing their energy in the form of a wind which behaves like a plasma-dominated MHD flow, at least far from the pulsar, composed primarily of electrons and positrons by number but with the energy flux largely carried by a numerically small population of heavy ions. As controversial and model dependent as that sounds, I will use this conclusion to sort among the proposed magnetospheric models.

\section{High frequency photon emission-relativis- tic particles?}

High frequency pulsed photon emission, mostly of $\mathrm{X}$ - and gamma-rays, form the next constraints in energetic order on magnetospheric models. In most cases, the observations provide only upper limits, but these can be powerful restraints on many of the proposed models. The observation of pulsed $\gamma$-rays from the Crab and Vela pulsars shows that in at least these systems, ultrarelativistic particle acceleration occurs, with efficiency of conversion of spindown energy loss to hard photon emission on the order of $1 \%$.

\section{Large voltages}

One expects to look for pulsed high energy photons (optical emission and/or X-rays and/or gamma-rays) first of all because they have been seen in a few cases (the Crab and Vela pulsars and PSR 0540-69 in the LMC are the most prominent examples), and secondly because the rotating magnetic field should generate huge voltages that could accelerate ultrahigh energy particles (Deutsch 1955). It helps to adopt a theoretical simplification in thinking about magnetospheric electric fields. Suppose the star is a good conductor with negligible interior circulation (both are excellent approximations in the present context). Neglect fluctuations shorter than the rotation time scale, so that we can model the system as steady in the corotating frame. Then there is a potential $\Phi$ such that the interior electric field is

$$
E=-\frac{\Omega \times r}{c} \times B-\nabla \Phi .
$$

This electric field requires the presence of a distributed space charge $\eta_{\mathrm{R}}=-\Omega \cdot B / 2 \pi c+$ relativistic corrections, and usually requires a net 
charge on the star. For example, a uniform interior field aligned with the rotation axis has uniform charge density and a net charge inside the star of $Q_{\mathrm{u}}=-\Omega \mu_{\mathrm{eff}} / 3 c$, with $\mu_{\mathrm{eff}} \equiv B_{*} R_{*}^{3}$, while a star centered, aligned point dipole has a central charge of $Q_{\mathrm{d}}=\Omega \mu / 2 c$ and a quadrupolar space charge. Inside the star, $\Phi=0$ to a very high degree of accuracy. Rotating a conducting star with a large scale surface magnetic field $B_{*}$ in vacuum produces external electric fields with a voltage drop from rotation pole to equator as measured in the corotating frame of $\Phi_{\text {vac }} \approx\left(\Omega R_{*} / c\right) B_{*} R_{*} \sim 6 \times 10^{16} \mu_{30} / P$ Volts, where $\mu_{30}=\mu / 10^{30}$ cgs. Perhaps a more relevant estimate is that introduced by Goldreich and Julian (1969), who assumed that plasma could fill the closed region of a dipolar magnetosphere, those field lines of the dipole which close inside $R_{\mathrm{L}}$, until $\Phi=0$ here too-in essence, the "star" extends to the light cylinder, within the closed zone. Then only the polar region, identified as those field lines of the undistorted dipole which would have extended beyond the light cylinder, can be the site of acceleration which can tap much of $\Phi_{\text {vac }}$. If the polar region is empty, the vacuum electric field above the cap yields a voltage

$$
\Phi_{\text {cap }} \sim \frac{\Omega^{2} \mu}{c^{2}} \approx 10^{13} \frac{\mu_{30}}{P^{2}} \text { Volts. }
$$

Furthermore, the vacuum field has as much electric field parallel to $B$ as points across $B$, so charged particles which got into such a field would be expected to accelerate to energies where gamma-ray emission is likely. Goldreich and Julian also properly observed that this vacuum electric field would be expected to pull charges from the surface until the polar regions of the magnetosphere also reach the same potential as inside the star, which opens the question of just how much of the vacuum polar cap voltage is delivered to particles which efficiently radiate, and also where this voltage appears, if it ever does. Goldreich and Julian suggested the extraction of charges corresponds to $E \cdot B$ being negligibly small, with an outflowing charge-separated current flowing off to the surrounding interstellar medium with $q \Phi$ perhaps no larger than the gravitational energy/particle. In their picture, the full energy of the polar cap potential drop is not delivered until a somewhat magical current loop is closed in the surrounding nebula or interstellar medium. In this zeroth order view, the pulsar dog does not bark at all, at least in pulsed gamma rays.

\section{$\gamma$-ray limits}

Nevertheless, the fact that a few pulsars do emit pulsed, observable, high energy photons, as well as the large polar cap voltages, has motivated many observers to seek pulsed $\boldsymbol{\gamma}$-rays, since particles a celerating along polar field lines form a good cal didate for lighthouse emission from neutron star The most useful observations came from studies . $50-\mathrm{MeV}$ to $2-\mathrm{GeV}$ photons by the SAS-2 (Ögelma et al. 1976, Thompson and Kniffen 1989)and COS(Buccheri et al. 1983) satellites, and by the HEAC A satellite in the low energy gamma-ray/hard X-ra regime, $15 \mathrm{keV}-2 \mathrm{MeV}$ (Knight et al. 1982). Tr Crab pulsar is detected in all these bands, the Ve pulsar only above $50 \mathrm{MeV}$, and useful limits were si on a number of others. For energetic purposes, it most useful to phrase these limits as fractions of tr spindown luminosity, $\eta=L_{\gamma+\mathrm{x}} / \dot{E}_{\mathrm{R}}$. "Detection: with $\eta>1$ have all been shown to be attributab to noise, while upper limits which find $\eta>1$ are ol viously not helpful. Table 1 shows the useful limit from objects selected because of their spatial pro: imity and their large spin-down luminosities. $\mathrm{Tl}$ entries are all upper limits, except for the Crab ar Vela, where the upper limits are marked explicit. and the other entries are clear detections.

Table 1 Fractional hard photon luminosity o: radio pulsars

\begin{tabular}{|c|rrr}
\hline Object & $15-175 \mathrm{keV}$ & $1-11 \mathrm{MeV}$ & $50-2000 \mathrm{MeV}$ \\
\hline Crab & 0.002 & 0.002 & 0.004 \\
Vela & $<0.000008$ & $<0.01$ & 0.01 \\
$0740-20$ & 0.008 & & 0.2 \\
$1055-52$ & 0.007 & 0.026 & 0.5 \\
$1929+10$ & 0.005 & 0.016 & 0.03 \\
$0540+23$ & 0.14 & & \\
$0950+08$ & 0.009 & & 0.16 \\
$0611+22$ & 0.07 & & \\
$1642-03$ & 0.018 & 0.3 & \\
$1822-09$ & 0.05 & & 0.006 \\
$1951+32$ & & & (historical) \\
(CTB 80) & & & \\
\hline
\end{tabular}

There are many caveats surrounding these nur. bers, including the uncertainties in the mome: of inertia $\left(10^{45} \mathrm{cgs}\right.$ was assumed), the amount beaming ( $\pi / 2$ ster was assumed), the photon spe trum assumed to convert photon counts to $\mathrm{e}$ ? ergy fluxes $\left(\varepsilon^{-2}\right.$ was used), and the long bac extrapolation of the period of PSR 1951+32 need to reanalyze the SAS-2 data. Nevertheless, it clear that most of a pulsar's energy is not goir into hard $\gamma$-rays, and any candidate model mu not turn the pulsar into a $\gamma$-ray dog, in spite of $t]$ enormous energies per particle implied by the lari voltages available.

\section{Soft X-ray limits on surface emission}

Many attempts have been made to detect radio pr sars with $\mathrm{X}$-ray telescopes sensitive to photons $\mathrm{b}$ 
tween a few hundred $\mathrm{eV}$ to $15-20 \mathrm{KeV}$. Much of the effort has been motivated by the search for the thermal emission from the whole neutron star, as it cools from its initial violent birth. Einstein observations (Cheng and Helfand 1983, Helfand 1984) have shown that eight radio pulsars (out of two dozen surveyed) with $\dot{E}_{\mathrm{R}}>10^{33} \mathrm{erg} / \mathrm{s}$ have unpulsed emission in the Einstein IPC band $\varepsilon<3 \mathrm{keV}$.

Table 2 Fractional unpulsed soft X-ray luminosity of radio pulsars

\begin{tabular}{|c|rrrr|}
\hline Object & $L_{\mathrm{X}} / L_{\odot}$ & $\dot{E}_{\mathrm{R}} / L_{\odot}$ & $L_{\mathrm{X}} / \tilde{E}_{\mathrm{R}}$ & $P / \dot{P}(\mathrm{yr})$ \\
\hline Crab & 12500 & 230000 & 0.05 & $10^{3}$ \\
MSH15-52 & 100 & 10000 & 0.01 & $2 \times 10^{3}$ \\
Vela & 0.5 & 3500 & 0.002 & $10^{4}$ \\
CTB80 & 0.4 & & & \\
$1055-52$ & 0.2 & 15 & 0.01 & $5 \times 10^{5}$ \\
$0355+54$ & 0.05 & 23 & 0.002 & $6 \times 10^{5}$ \\
$1642-03$ & 0.03 & 0.5 & 0.06 & $3 \times 10^{6}$ \\
\hline
\end{tabular}

Typically, $\sim 1 \%$ of the rotational energy loss goes into the unpulsed X-rays. The Crab Nebula also radiates about $5 \%$ of $\dot{E}_{\mathrm{R}}$ into the Einstein band, which also supports the nebular hypothesis. If all pulsars put a few per cent of their spindown energy into X-ray emission within the Einstein band, this survey found almost every object that it could have, given its sensitivity limit. Thus, one can firmly conclude that there is no pulsed, soft X-ray emission from essentially all these objects, at the level of $1 \%$ of the total energy loss. Magnetospheric models, most of which focus on particle acceleration activity in the polar flux tube and the outer magnetosphere, must not supply large amounts of energy in the form of relativistic particles or high energy photons precipitating onto the magnetic poles, since the energy is deposited at depths greater than 100 $\mathrm{g} / \mathrm{cm}^{2}$, where the dense matter of the star can thermalize the incident flux and re-radiate it as soft $\mathrm{X}$ rays. The pulsed fraction in the Einstein observations is certainly less than $50 \%$, and in some cases less than $10 \%$. Therefore, the total thermal emission from each polar cap is less than $10^{31} \mathrm{ergs} / \mathrm{s}$, corresponding to a polar-cap temperature of less than $800 P^{-1 / 4} \mathrm{eV}$. The upper limits on the total emission from the rest of the stars in the survey give similar limits on polar-cap temperatures.

\section{Surface conditions - theory}

The ability of the stellar surface to emit particles freely turns out to be an essential aspect of magnetospheric models. Nothing is known about this experimentally, since we have no photons clearly coming from the surface whose spectra could tell us about surface conditions, nor are the physical states of the surface matter accessible to laboratory investigation. Müller (1984), Jones (1986) and Neuhauser et al. (1987) have shown that iron, the most likely constituent of the stellar surface, does not form a bound lattice on the surface of a strongly magnetized neutron star. These authors used three different techniques to estimate the binding energy of iron atoms at the surface, all of which yield the result that iron has its lowest energy state as unbound atoms rather than the chains required if the surface is to have a non-zero work function. The results of Jones and of Neuhauser et al. agree to within $2 \%$, although they are found by quite different methods. Previous calculations, summarized by Flowers et al. (1977), suggested iron is bound into a solid crust, with sufficient binding energy to be able to resist extraction even by the electric field of the vacuum. These calculations are known to contain errors (Müller 1984). The more recent work looks to be sufficiently reliable that one should treat free emission from the surface as a fact, not a theory, both for geometries in which $\boldsymbol{\Omega} \cdot \boldsymbol{B}<0$ over the polar cap (the case of ion emission), as well as for the case $\boldsymbol{\Omega} \cdot \boldsymbol{B}>0$ (electron emission from the polar cap), where all estimates indicate that electrons are freely available from the star. Thus, vacuum regions in the magnetosphere (if any) reach the surface only on a set of measure zero, and all models of particle acceleration at and above the poles must not depend on finite binding in the surface.

\section{Radio emission}

\section{Polarization}

It is notoriously difficult to derive physical information from the radio emission. However, the rotating single vector model for pulsar polarization phenomenology, with the vector interpreted as the projection onto the sky of the polar field lines of a rotating dipole field (Radhakrishnan and Cooke 1969), has proven so robust in the face of an ever increasing data base (Lyne and Manchester 1988) that one should, I think, take seriously the idea that pulsar radiation really does come from the relatively low altitude polar field lines, with beaming parallel to the magnetic field. Note that this sense of beaming is not a priori essential to the rotating vector model; one could have beaming across the magnetic field at low altitude, as in planetary collective radio emission, and still satisfy the kinematic constraints of the polarization variations observed. I accept the standard variation of the lighthouse model because it fits in with plausible physical ideas about how the radiation is formed. 


\section{Low altitude-scintillation constraints}

Several recent experiments have made clever use of interstellar scintillation to set constraints on the dimensions (Cordes, Weisberg, and Boriakoff 1983, Smirnova and Shishov 1989). These authors show that the size of the emission region is not greater than $\sim 100 \mathrm{~km}$, based on the existence of scintillations appearing in the signals from several pulsars with double profiles. The idea is that the double peaks in the waveform come from physically separated emission sites. If these sites are too widely separated, the emission will not show scintillation, having been resolved by the interstellar "lenses" formed by electron-density fluctuations. If one assumes the components of the pulse profile form at the boundary of a polar flux tube whose surface opening angle is $\sim(\Omega R / c)^{1 / 2}$, then the spatial separation of the components is $\sim 10(\Omega r / c)^{3 / 2} \mathrm{~km}$ at radius $r$. The observations then limit the height of the apparent radio "photosphere" to be less than $10 \%$ of the light-cylinder distance. Note that this conclusion can be avoided even in dipole geometry if the components do not form near the boundary of the polar flux tube, but since $I$ suspect emission is closely associated with the boundary layer at the edge of a stream of plasma flowing out on open field lines (Arons 1979, Arons 1981a), I think one should take seriously the limitation of the emission region to altitudes of $1000 \mathrm{~km}$ or less.

\section{Collective emission-dense plasma}

These kinematic constraints say nothing about the properties of the emitting medium, however. "All" one has to do is to arrange for a plasma maser or travelling wave amplifier to exist at low altitude, with amplification preferentially along the magnetic field. Under the strong field circumstances inferred from the spindown theory, such an amplifier must have its plasma frequency somewhere in the radio domain. The main options for collective emission processes involve either some sort of cyclotron or synchrotron maser, or some form of collective excitation at the plasma resonance. The former class of processes is ruled out by the high magnetic fieldthe cyclotron frequency is too high, and the time for particles to lose their gyrational momenta is less than the time for any mechanism to keep them in excited gyrational states. The latter class requires a relatively dense plasma - with $\omega_{\mathrm{p}} \sim 10^{9}-10^{10} \mathrm{~s}^{-1}$, a static plasma with electron (or positron) densities in the range $\sim 10^{7}-10^{9} \mathrm{~cm}^{-3}$ would be needed. Indeed, if the broad band observed spectra are to be produced by narrow band emission at the plasma frequency, this whole range of densities must occur. Interestingly, the number density corresponding to the co-rotation charge density falls in this range at altitudes $\sim 100-1000 \mathrm{~km}$, consistent with the radii suggested by the scintillation constraints.

To go beyond this point requires an explicit knowledge of the emission mechanism. Many people believe in "bunched coherent curvature" radiation as the emission mechanism, because it may simultaneously explain the strong beaming along $B$, the formation of the high brightness temperature in the emission, and the organization of the beaming pattern into the observed multiple cone geometry. While this is not the only interesting possibility (e.g., Arons 1981b), I will adopt it here because it illustrates how one can use the elementary ideas to put constraints on possible magnetospheric models. In its naive form (e.g., Benford and Buschauer 1977), one simply assumes the emission from particles moving relativistically along the curved polar field lines is that of a single particle in vacuum, enhanced by some bunching mechanism which crowds many particles into one vacuum wavelength, or even creates a phased array, and makes them radiate together. Phrased this way, one needs a plasma moving outwards with Lorentz factors on the order of several hundred to $\sim 1000$, in order to create waves in the meter to centimeter range regime. To be collective, however, the relativistic plasma frequency appropriate to bunching motions parallel to the particles' momenta, $\omega_{\mathrm{pr}}=\left(4 \pi e^{2} n_{\mathrm{e}} / m_{ \pm} \gamma^{3}\right)^{1 / 2}$, must also be in the same frequency regime. This has two consequences. The first is that the plasma must be a whole lot denser than the basic corotation density - something has to work to raise the density above what would be lifted off the star by the magnetospheric electric field alone. If any mechanism involving parallel response of a relativistically flowing plasma at the plasma frequency is to describe the collective emission, then

$$
\begin{aligned}
\frac{e N}{\eta_{\mathrm{R}}} & >\frac{1}{2} \frac{\omega^{2}}{\Omega \omega_{\mathrm{c}}} \gamma^{3} \\
& \sim 5 \times 10^{3}\left(\frac{\omega}{1 \mathrm{GHz}}\right)^{2} P B_{12}^{-1}\left(\frac{r_{\mathrm{em}}}{R_{*}} \frac{\gamma}{100}\right)^{3} .
\end{aligned}
$$

Here $r_{\mathrm{em}}$ is the emission radius. If we also assume the emission has something to do with vacuum curvature radiation, we have $\gamma^{3} \sim \omega \rho / c$, where $\rho \sim R_{*}\left(c / \Omega R_{*}\right)^{1 / 2}\left(r / R_{*}\right)^{1 / 2}$ is the radius of curvature in a dipole field. For any form of collective curvature radiation in a dipole field, the density limit then becomes

$$
\frac{e N}{\eta_{\mathrm{R}}}>\frac{1}{2} \frac{\omega^{3} \rho}{\Omega \omega_{\mathrm{c}} c} \sim 2 \times 10^{5}\left(\frac{\omega}{1 \mathrm{GHz}}\right)^{3} \frac{P^{\frac{3}{2}}}{B_{12}}\left(\frac{\tau_{\mathrm{em}}}{R_{*}}\right)^{\frac{1}{2}}
$$

However, the second consequence of the large plasma frequency is that the Razin effect suppresses 
the vacuum emission (Arons and Barnard 1986). Therefore, the simple relation $\omega \sim c \gamma^{3} / \dot{\rho}$, which depends on the relation between retarded time and observer's time for waves propagating in the vacuum, is no longer correct, and the naive idea of simply multiplying the vacuum emission rate by a bunching factor is just wrong. Instead, one looks for mixed electrostatic-electromagnetic modes of the derse plasma (Asséo, Pellat, and Sol 1983, Beskin, Gurevich, and Istomin 1988c), which can either smoothly change to electromagnetic waves which propagate to infinity, or which can couple to escaping waves at higher altitude (Barnard and Arons 1986, Beskin, Gurevich, and Istomin 1988c). Thus, the basic limit on the density is eq.(6), set simply by requiring the plasma frequency to exceed the frequency of the observed radio emission. All the radio emission models now under serious investigation satisfy this constraint. Fortunately, a number of models of pair creation at and above the polar cap supply dense outflowing plasmas with parameters in this regime, as is discussed below.

\section{Current systems}

Flinging a dense plasma out at the speed of light almost certainly involves a current flow on the polar field lines. If the plasma is composed of more than one species, with density very large compared to $\eta_{\mathrm{R}} / q$, then the plasma easily adjusts the relative densities of the species to keep the charge density just about equal to $\eta_{R}$, through weak "ambipolar" electric fields along $B$ which speed up one species and slow down the other (Scharlemann 1974; Cheng and Ruderman 1977b, Arons 1981b, Beskin, Gurevich, and Istomin 1988c). In principle, this plasma flow could occur without current flow. In fact, all the suggested mechanisms for making an outwardly bound relativistic plasma on polar field lines involve some current flow, most with current density $\sim c \eta_{R}$ assumed in the model. If this dense plasma arrives in the outer magnetosphere and can be accelerated to energy densities even remotely comparable to the electromagnetic energy density, strong currents form to map this stress back down to the neutron star. The problem of current flows has been the central preoccupation of "formal" pulsar theory for the last 20 years. I now turn to a brief review of the ideas (many) and results (not so many) on the global magnetospheric structure, from the perspective of making an observationally relevant model which fits the various constraints outlined here. Along the way, we will find that most possibilities for the origin of the radio emission involve some variation of the passage of the current through the plasma as the source of the free energy that excites the radio emission.

\section{Current flow and pair cre- ation}

'A hypothesis or theory is clear, decisive and positive but is believed by no one but its author. Experimental findings, on the other hand, are messy, inexact things which are believed by everyone except those who found them.'

Attributed to Harlow Shapley

The generic problem of a pulsar magnetosphere model with observational relevance therefore is 'how does the magnetized neutron star produce a relativistically outflowing plasma on polar field lines, consistent with free extraction of plasma from the stellar surface $\left[(E \cdot B)_{r=R_{0}} \approx 0\right]$ and with radiatively quiet spindown of the star at frequencies above the radio?' Many answers have been suggested to this question; I will review only those that appear to me to have some relevance. The basic problem faced by the magnetospheric theorist is, how does the magnetosphere fill, if plasma is supplied by electrical extraction from the stellar surface but the magnetic field is everywhere the dominant energy density? The problem is illustrated by the cartoon (essentially that of Goldreich and Julian) in figure 1 , in which I suppose the magnetosphere is filled, and has an electric field approximately equal to its co-rotation value everywhere. I have drawn

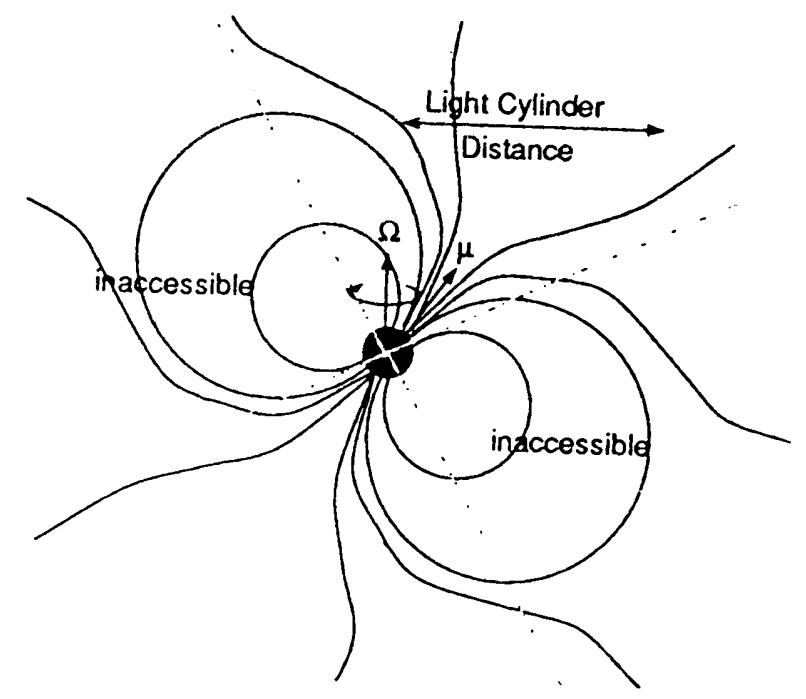

Figure 1 Accessible and inaccessible regions of a rotating magnetosphere supplied by non-neutral plasms extracted electrically from the stellar surface.

the figure as if there is such a thing as open field lines-if all the field lines are closed, the same problems exist. If the electric field can freely extract charges from the surface, pulling particles out whose sign of charge is the same as that of $\eta_{R}$, but there is no other source of plasma, field lines which cross the 
"null surface" where $\boldsymbol{\Omega} \cdot \boldsymbol{B}=\mathbf{0}$ pass into a region where the star cannot supply the charges required, by motions strictly along $B$. As was first pointed out by Holloway (1973), the filled model pictured is unstable to emptying out, leaving vacuum "gaps" in the magnetosphere.

Most papers reporting results on these questions are extremely technical. By contrast, I will restrict myself to the cartoon approximation, in the interests of drawing out the physical ideas.

Theorists' responses to Holloway's criticism of the filled model can be divided into two general camps. The first group takes the charge separated model seriously and attempts to find either static models or models with current flow which are consistent with the existence of the inaccessible zones shown above. These people have tended to regard pair creation as an interesting adornment to their models, but not as something which fundamentally alters the current-causing stresses which depend upon vacuum-like electric fields in the outer magnetosphere, a possibility which requires the null surfaces to have major electrodynamic significance. The second camp has followed the thought that the extraction of a non-neutral current at the surface occurs with sufficient vigor to lead to the creation of a dense pair plasma which flows out and fills the whole magnetosphere. Since the density of the pairs is large compared to $\eta_{\mathrm{R}} / e$, the adjustment to the required charge density requires a (relatively) trivial adjustment of the plasma components' flow velocity as the null surfaces are approached and crossed. Theorists of this school have assumed and worked on models in which the energization of the dense plasma in the outer magnetosphere leads to a global current system which is consistent with the surface extraction of current with pair creation just above the surface, with outer magnetospheric electric fields which are not dependent on the existence of large scale vacuum zones. Therefore, pairs are regarded as being of fundamental importance to determining the qualitative character of the magnetospheric dynamics. I more or less subscribe to this second school's beliefs, as a working hypothesis, although in earlier years I held somewhat mixed views. I emphasize that no matter how forcefully stated, views on either side of this divide are only working hypotheses. Neither tribe has yet to demonstrate a working, quantitative model in any geometry which derives from either view and which satisfies all the constraints outlined in the previous sections. As a result, we do not even know which observations might decide between these qualitatively distinct starting points.

\section{Current free models}

Goldreich and Julian's ideas included not only a filled magnetosphere, but also the thought that currents would flow on open field lines, creating the $B_{\phi}$ needed to produce the observed torque. The hope of phenomenologically minded people is to somehow tap the energy associated with this polar current flow to create the polar lighthouse. The obvious difficulty is, if the only way to supply the charges is the pull of the magnetospheric electric field, what stops the star from charging up ${ }^{2}$ ? Answering this question is essentially the same as figuring out what supplies charges of the sign opposite to those drawn from the polar regions to the "equatorial" zone between the null surfaces. Goldreich and Julian hypothesize "hanging charge clouds" (their language) with the same sign as the sign as $\eta_{R}$, whose electrical pull would overcome the residual effects of the vacuum electric field and suck out the required return currents (and, although they did not say so explicitly, send the required oppositely signed charges into the regions where field lines cut the null surfaces). These currents were supposed to close somewhere far outside the light cylinder. Models of static charge clouds satisfying these requirements have never been constructed.

What has been suggested, however, and to some extent constructed, is a class of static models which does satisfy $\left.E \cdot B\right|_{r=R_{*}} \approx 0$ and avoid the problem of filling the region between the null surfaces. Michel (1982) suggested, and Krause-Pohlstorff and Michel $(1985 \mathrm{a}, \mathrm{b})$ crudely constructed, models in which charges are lifted off the surface by the electric field, fill a dome over the polar caps with charges of the same sign as $\eta_{R}$ over the poles, while in the equatorial region composed of field lines which never penetrate the null surface, the electric field pulls out charges of sign opposite to that of the polar dome, to fill an equatorial "disk".

This will work if there exists a surface of the polar dome and of the disk where $E \cdot B=0$ again, and beyond which the magnetosphere is a vacuum - a large scale vacuum gap is accepted, and in fact, most of the magnetosphere is empty, with the plasma domes and disk extending only a few stellar radii up, if the stellar charge differs from the filled magnetosphere value by amounts of order $Q_{\mathrm{d}}$ itself. This gap never reaches the surface (actually, it contracts to a line at the surface). Within the plasma dome and disk, the particles are at rest, if they have no thermal dispersion at all; gravity and electric force are exactly balanced. If the small thermal effects are included, the electric potential simply adjusts to trap them-see Fawley (1978), who

\footnotetext{
${ }^{2}$ More precisely, what keeps the star charged at the value expected for the filled magnetosphere?
} 


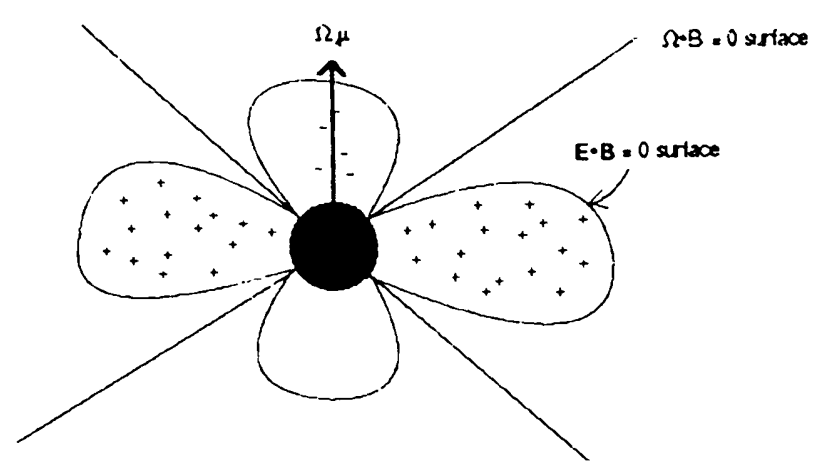

Figure 2 Static magnetosphere proposed by Michel. Trapped plasma yields $E_{\|}=0$ on the stellar surface and on a free surface of the plasma at altitudes well inside the light cylinder, when the star has a net charge differing significantly from that of the Goldreich-Julian model.

showed (in a somewhat different context) that such a plasma boundary could be constructed on the polar field lines which bend away from the magnetic axis of the oblique rotator. The numerical calculations used to construct examples of these dead magnetospheres are rather crude, and more work on such dead structures is in order, particularly since they may well be of interest for the $\gamma$-ray burst sources. The price paid by this kind of a model is an alteration of the total charge on the star from the value expected when the magnetosphere is filled. If the magnetic field in the stellar interior is well described by a point dipole, the charge associated with a filled magnetosphere is $Q_{\mathrm{d}}=\Omega \mu \cos \chi / 2 c$. In the absence of pair creation, there is no reason in principle against having an altered charge--it all depends on whether the star would exchange charges with the interstellar medium. One argument against such exchange is the expulsion force exerted by vacuum dipole waves on charged particles from the interstellar medium (Arons and Barnard 1983, Michel 1987a). This force prevents all but the highest energy cosmic rays from getting into the magnetosphere. Interstellar grains may succeed in entering, however (Cheng 1985), although their entry affects the state of the magnetosphere only if the system does not have a dense plasma outflow on the polar field lines.

However, as a model of an active pulsar, this picture is not much help. The dome and disk end deep within the magnetosphere. Therefore the torque must be that of the vacuum rotator, in oblique geometry - there is no outflowing wind at all, never mind one composed of $e^{ \pm}$and ions, nor is there a dense relativistic plasma outflow on polar field lines. For most relevant rotation rates, however, the vacuum zones are unstable to pair creationstray $\gamma$-rays from the background will trigger $10^{20}$ avalanches/sec. If the gap could be maintained, plasma would shower down on the surface with an energy flux in relativistic particles comparable to the spin down power and broadcast a $\gamma$-ray luminosity to infinity with about the same luminosity, unacceptable consequences for models of normal pulsars ${ }^{3}$. However, as a model for neutron stars beyond the pulsar death line, where voltages are too small to cause electromagnetic cascades to form, the dome and disk model may be on the right track, and might turn out to be useful both as the basic input for models of $\gamma$-ray bursters. To make an active magnetosphere, however, requires some transport of dense plasma, and all schemes for doing this require some form of conduction current flow.

\section{Current flow without pairs-closed currents}

Why should currents flow in a magnetosphere? There is only one reason-stress on the magnetic field. These stresses probably must occur at distances not much beyond the light cylindercertainly, if the wind model for nebular excitation is correct, stress in the distant interstellar medium cannot be communicated back to the tiny magnetosphere.

If there is no pair creation in the magnetosphere, the density must be on the order of $\eta_{\mathrm{R}} / q$, while the energy/particle cannot exceed $q \Phi_{\text {cap }}$. Thus, the maximum energy density possible in charge separated plasma is

$$
U_{\max }=\Phi_{\text {cap }} \eta_{\mathrm{R}} \approx \frac{B\left(R_{\mathrm{L}}\right) B(r)}{2 \pi} ;
$$

plasma stress can induce current only at distances comparable to the light cylinder. A whole collection of different schemes, all related, have been advanced which have attempted to take advantage of this point to create a magnetospheric model in which the current flow closes in the outer magnetosphere.

The earliest of these suggestions is due to Jackson $(1976,1980)$, who first pointed out the possible existence of the plasma dome and disk. He also observed that the electric field in the outer magnetosphere might exceed the magnetic field, and

\footnotetext{
${ }^{3}$ Michel (1990) has recently suggested just this sort of model, in which the radio emission comes the electromagnetic fields associated with the narrow confinement of the pair discharges in the direction along the magnetic field. In my opinion, he has not paid sufficient attention to the fate of most of the energy in his model, which is in the dissipation of the discharge energy in $\gamma$-ray emission and thermal radiation from the neutron star, both with luminosities comparable to $I \Omega \dot{\Omega}$.
} 


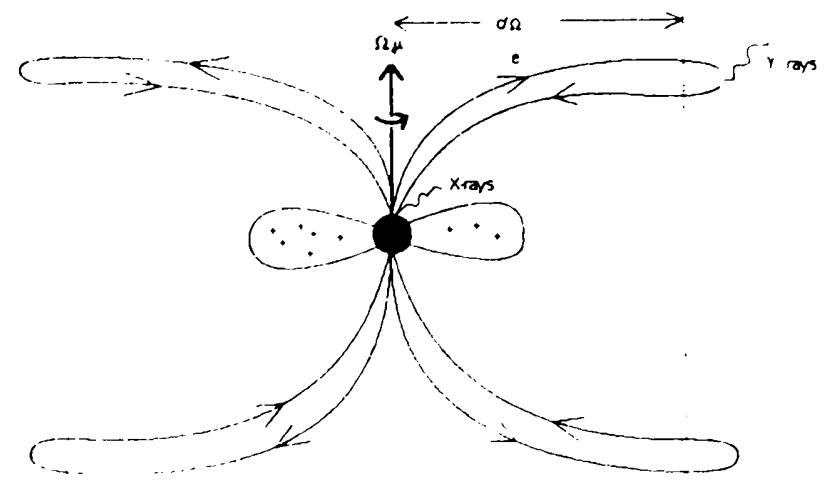

Figure 3 Closed current flow pattern in magnetospheric models based on charge separated outflow without pairs.

pointed to the possible acceleration in this region as the origin of the transverse current flow. Rylov (1977; see also Rylov 1989 and references therein) and Mestel et al. (1985 and references therein) have advocated the same origin for the magnetospheric stress. If the current flow can be established, these various models show that particles get highly accelerated near the light cylinder, with precipitation of the same particles back to the stellar surface at lower magnetic latitudes. Most of these models rely upon having non-relativistic flow velocities parallel to the magnetic field on the outbound part of the current loop. They all have an ion region near the magnetic equator similar to the disk in the Pohlstorff-Michel model, and they either have downflow of highly energetic particles onto the stellar surface, with precipitation energy put into the star comparable to the whole energy loss observed in spindown, or they expend most of their energy as curvature $\gamma$-rays from the acceleration zone itself, with typical photon energies in the $100-\mathrm{MeV}$ to $\mathrm{GeV}$ range.

In the precipitation case, the currents clearly do not have much to do with spindown, since a completely closed current system does not support a toroidal field far from the star, and spindown of an oblique rotator would depend on some modified form of a vacuum wave. Thus the main energetic motivation for a magnetospheric model is lost, and the current flow is an embarrassment, since comparable amounts of energy are put into heating the stellar surface. So far as I can make out, Rylov's and Jackson's forms of these ideas are certainly excluded by the surface X-ray emission limits of the younger pulsars. In contrast, the model described by Mestel $e t$ al. converts most of the acceleration power into $\gamma$-rays, at least in the shorter period objects. This has the advantage of allowing even an aligned rotator to spin down, but at the cost of putting essentially all the spin down energy into a curvature spectrum of $\gamma$-rays radiated into most of the sky which would have been easily observable from the short period pulsars studied by SAS-2, COS-B and HEAO-A. Furthermore, none of these schemes provides the dense wind desirable for plerion excitation. I conclude that these models are also not about real pulsars. They do show, however, the importance of strong acceleration in the outer magnetosphere, through the influence of large-scale, vacuum-like electric fields, and they show that current closure is possible, even in a charge separated medium. My own suspicion, however, is that a charge separated magnetosphere would just shut off, since if one were to imagine a downward excursion of the current, particles refilling the magnetosphere could simply fill in the dome and disk and leave the outer magnetosphere as a vacuum. In any case, there is now wide consensus that if pair creation is absent, the magnetospheric morphology is basically closed.

\section{Current flow with pairs}

The closed current, pair-free models, while revealing important questions of principle, do not satisfy our phenomenologically driven need to have a dense plasma streaming out along polar field lines, with the torque finally carried by a wind with an energetically significant component of heavy ions. I now turn to ideas and calculations which have a better chance of fulfilling these requirements.

\section{Pair creation-low altitude and open circuits}

The only way known to get a dense plasma moving out relativistically on polar field lines is through pair creation over the polar caps. Sturrock (1971) did the ground-breaking work on this, with a model that does satisfy the free surface emission boundary condition. As is well known, the basic idea is the acceleration of particles in $E_{\|}$, the emission of curvature $\gamma$-rays and their conversion into pairs in the strong magnetic field, features which have survived in every subsequent model. However, his model of $E_{\|}$is flawed. While he does satisfy $E \cdot B=0$ at the surface, he made no attempt to include the effect of the pairs created on the accelerating electric field. As a result, his scheme results in the whole polar cap potential drop being applied to the extracted current within a height comparable to the polar cap width. The same potential drop is applied to all the pairs formed also. Since the pairs are born with initial energies much lower than the potential energy drop in the flow, charges of sign opposite to $\eta_{\mathrm{R}}$ are accelerated back down to the surface, with a current density at least $c \eta_{R}$. Therefore, the energy flux on the surface is $>c \eta_{R} \Phi_{\text {cap }}$, which results in the whole polar cap being heated with more than 
the spin-down luminosity.

Arons and Scharlemann (1979) and Arons (1983a) first showed how low altitude pair creation might work, in a locally self-consistent manner. Their model builds on some aspects of charge separated, pair-free current flow at low altitude previously discussed by Scharlemann et al. (1978). These authors assumed the surface freely emits charges, which are assumed to be extracted in the form of a relativistic, non-neutral current flow at low altitude - the acceleration to relativistic energies happens within a few centimeters of the surface (Michel 1974, Fawley, Arons, and Scharlemann 1977). This kind of current flow has a unique current density - since the velocity is essentially $c$ everywhere, the charge density and current density have a constant ratio. If the charge density is fixed at the surface, so is the current density. Free emission at the surface requires $E: B=0$ at the surface, which in turn demands a unique charge density, such that the charge density of the beam at the surface is (almost) exactly equal to $\eta_{\mathrm{R}}\left(R_{*}\right) \propto B_{\|}\left(R_{*}\right)$, where $B_{\|}$is the component of $B$ parallel to the rotation axis. Such relativistic acceleration of a completely non-neutral beam is possible only on so-called "favorably curved" field lines, those which bend toward the rotation axis (Scharlemann, Arons, and Fawley 1978), if the effect of general relativity is ignored. These exist only in the oblique rotator. The reason is that the real charge density of the beam drops with height in proportion to $B(r) / B_{\|}\left(R_{*}\right)$, for relativistic flow along field lines, while $\eta_{\mathbf{R}}$, the charge density required to short out $E_{\|}$, drops in proportion to $B_{\|}(r) / B_{\|}\left(R_{*}\right)$. On polar field lines which bend toward the rotation axis, the real charge density of the assumed beam is lower than $\eta_{R}$. As a result, a charge on the surface looks up and sees an effective total charge density of sign opposite to its own and is attracted up off of the surface-above the surface, the vacuum $E_{\|}$ is not quite shorted out, with voltage drops exceeding $10^{13} \mathrm{~V}$ appearing within heights less than $R_{*}$ [although usually exceeding $\varpi_{\text {cap }}=R_{*}\left(\Omega R_{*} / c\right)^{1 / 2}$ ] for neutron stars on the active side of the death line in the $P-\dot{P}$ diagram. The physics of this beam flow is very similar to that of a laboratory device known as a "foil-less diode" - see Ott et al. (1977) for a nice description of these gadgets. In the interests of verbal consistency, I will call this low altitude acceleration region a polar "gap", even though it is not the same as the vacuum gaps with $E \cdot B \neq 0$ at the surface advocated by other authors (Ruderman and Sutherland 1975, Gurevich and Istomin 1985b).

The opposite happens on field lines which curve away from the rotation axis (all of the polar field lines in the aligned rotator)-relativistic, timesteady, charge-separated current flow composed of outwardly flowing charges is impossible, in the aligned rotator, as is explicitly pointed out by Scharlemann et al. (1978). They also found, and briefly mentioned, that lower current solutions can exist, in which the beam flow over the polar caps occurs at nonrelativistic speed ${ }^{4}$ with a current density somewhat below $c \eta_{R}$, a point independently made and exploited in the attempts to construct the aligned rotator models with closed current loops described above-see Rylov (1979) and Mestel et al. (1979).

Arons and Scharlemann's model takes advantage of the high voltages developed in the beam flow on the favorably curved field lines of an oblique rotator to create a dense, outflowing pair plasma, using the same physics as Sturrock suggested. When the extracted beam is electrons $(\Omega \cdot B>0)$, the curvature gamma-rays emitted directly by the beam are absorbed and form pairs. The pairs form with initial energies on the order of a few $\mathrm{GeV}$, much smaller than the $\mathrm{TeV}$ potential energy drop through which the beam passes. Therefore, positrons are trapped and accelerated back down to the star, while the newly formed electrons are added to the outflow, until the $n_{e}$ increases to become equal to $-\eta_{\mathrm{R}} / e$, where the density is now sufficient to completely short out $E_{\|}$. At this surface above the star, which we called the "pair formation front" because almost all of the pair creation occurs above this well defined transition layer, $\boldsymbol{E} \cdot \boldsymbol{B}=\mathbf{0}$ again, and also $\partial E_{\|} / \partial s=0$, where $s$ is the distance along field lines - this condition is required if the acceleration region is to be joined smoothly to the dense plasma above, and replaces the simple condition of $n_{\mathrm{e}}=-\eta_{\mathrm{R}} / e$ since finite charge non-corotation charge density is needed to support the total perpendicular electric field. Since the beam charge density is everywhere close to, although slightly less than, $\eta_{R}$ in absolute magnitude, only a small number of pairs are created in the acceleration region, and only a small number of highly accelerated positrons bombards the surface, so the thermal X-ray emission from the cap is small (Arons 1981a). The emission calculated in this paper is still below detection by Einstein and by ROSAT, but it may be testable by the larger area detectors to be flown on Spectrum X- $\gamma$, XTE and especially AXAF, whose imaging capabilities will greatly reduce the back-

"These models fail to satisfy the phenomenological demand of relativistic outflow at low $\left[r<(0.1-0.01) R_{L}\right]$ altitude. In addition, they require either non-monotonic flow velocities and potentials, or (more likely) spatially trapped charges with non-monotonic potentials through which the current carrying charges flow. In both cases, the flow is subject to instabilities of the trapped particle variety, whose associated anomalous resistivity is more than sufficient to restore the voltage drop to relativistic values, making the slow flow solutions physically unrealizable. 
ground. The same ideas apply if $\boldsymbol{\Omega} \cdot \boldsymbol{B}<0$. Here, an ion beam is extracted from the surface, which does not itself radiate the curvature gamma rays. However, a few pairs are created as the ions accelerate off the surface, by (for example) conversion of thermal photons in the Coulomb fields of the accelerated ions (Ruderman 1981, Jones 1981). The positrons form a low density beam, which behaves just like the electron beam in the electron extraction case, so long as the number of pairs finally created at the top of the acceleration zone is large.

The dense plasma is formed above the pair formation front, since the accelerated electron beam continues to radiate convertible gamma rays. Various estimates and calculations of the multiplication factor (Tademaru 1973, Ruderman and Sutherland 1975, Arons 1981b, Daugherty and Harding 1982 , Gurevich and Istomin 1985b) all suggest that $\kappa \sim 10^{3}-10^{5}$ pairs are produced per primary radiating particle in the acceleration zone, with the higher multiplicities applying to short period, strong field objects, where the gap height is small. Especially when the geometry is appropriate to electron extraction, the resulting particle flux is sufficient to provide the dense plasma needed both for the radio emission and for the wind powering the external nebula.

Before going on to relate these ideas to global models, I emphasize that the ever popular vacuum gap ideas (e.g., Ruderman and Sutherland 1975, Gurevich and Istomin 1985) are excluded by the lack of binding in the crust. In addition, they violate the very small limits on polar cap X-ray emission, because they develop a high energy outward directed beam (positrons, with current density $\sim c \eta_{\mathrm{R}}$ in most models) and a high density pair plasma above the gap at the price of accelerating an equally high density beam (usually electrons) into the crust. The thermalization of this energy implies unacceptably large $\mathrm{X}$-ray emission. When applied to the Vela pulsar, for example, these RudermanSutherland style models yield polar cap luminosities 3 to 5 times the upper limit set by the Einstein observations.

The principle theoretical difficulty represented in the Arons and Scharlemann model is that it works only if the current density is very close to the Goldreich-Julian value, and it leaves open how one populates the outer magnetosphere with a dense plasma on the unfavorably curved field lines. Let me leave aside the question of the current density for the moment, since the second question raises interesting possibilities in its own right. If one takes seriously the inability of the star to supply a dense plasma to the same region which is inaccessible to charge separated flow, one has to take seriously the presence of large vacuum-like electric fields, as in the models of Rylov and Mestel et al., but now possibly confined by pair creation into "outer gaps" (Cheng and Ruderman 1977b). I show a cartoon I once published in another conference proceedings, (Arons 1983c), which is an attempt to think through qualitatively what might be a completely open-circuited model.

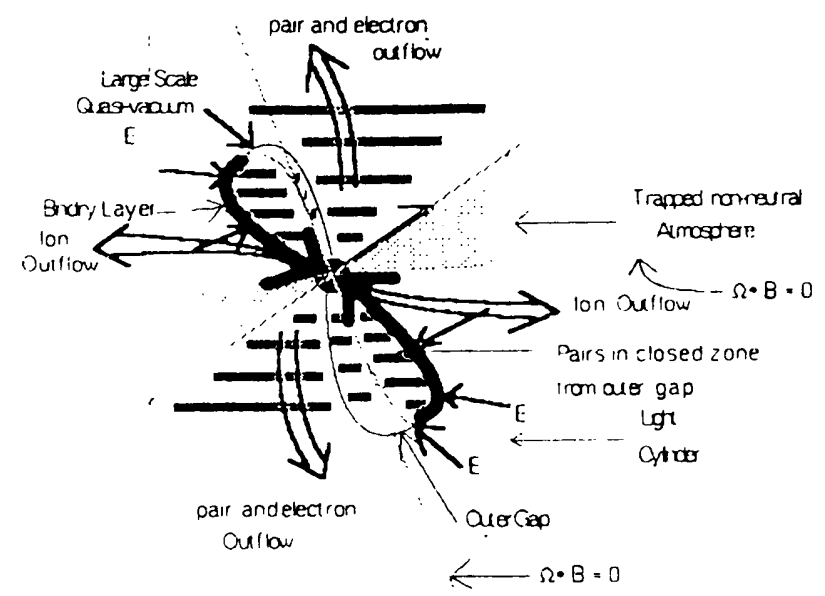

Figure 4 Cartoon model by Arons, mixing large outer gaps with polar outflow of pair plasma on unfavorably curved field lines.

The essential ideas are 1) a dense pair plasma generated according to the Arons and Scharlemann model flows out along the favorably curved open field lines; 2) a gap-like region opens up in the closed zone, whose pair production supplies the dense plasma assumed on closed field lines; 3) a trapped, charge separated atmosphere fills the unfavorably curved field lines between the polar caps and an $E \cdot B=0$ surface which forms close to the null surface; and 4) beyond the last closed field line in the formally inaccessible zone, there is a large region with $E>B$ with radial extent comparable to $R_{\mathrm{L}}$ itself. It was possible to show, within this picture, that the closed zone shields itself from the non-corotation external electric field by forming a boundary layer charge density and current, corresponding to the precipitation of a dense electron stream onto the edge of the polar cap. This precipitating stream forms a "dense charge cloud" above the polar cap which attracts up an ion stream that flows out and enters the large "vacuum" zone. If this large vacuum zone really has non-corotation electric field comparable to the total field, the ions carry a current comparable to the Goldreich-Julian current and are accelerated to an energy density comparable to $B^{2} / 4 \pi$, thus providing stress sufficient to drive the current circuit in the first place, and also providing an outflow current sufficient to balance the current present on the favorably curved field lines. The good features of the idea are a complete circuit, which closes only at infinity, with ions 
flowing out in the equatorial region, where we want them to power the Crab Nebula. Furthermore, the Larmor radii of the ions are comparable to $R_{\mathrm{L}}$, so the ions might leave most of the magnetic field lines behind, thus providing a second (historically, the first) explanation for the apparently low value of $\sigma$ in pulsar winds.

I never published the details of this scheme, because it contains the seeds of its own destruction. In order to create the dense precipitating plasma that pulls up the ions, one must fill the closed zone with pairs. Doing this with a gap on closed field lines leads to immediate trouble with the soft X-ray limit, since the potential dropped in the gap must be regained near the star in a relativistic double layer, whose acceleration of particles down to the surface creates too much thermal X-ray emission. The second problem is the lack of an obvious source of pairs in the equatorial flow to feed the surrounding nebula. This might be overcome by noting that pair avalanches can be triggered by the passage of ions through whatever thermal photons are around, but quick estimates led to problems with meeting the $\gamma$ ray emission limits. Finally, later developments by Cheng, Ho and Ruderman (1986a, b) showed that at least in the Crab and Vela pulsars, pair creation by particles accelerating along the open magnetic field lines might lead to pair formation with the radial extent of the outer gap limited to much less than is needed to give the ions an energy density comparable to the electromagnetic energy density, thus undermining the dynamical source of the current flow. Nevertheless, I think the idea of accelerating ions to high energy, which have been sucked up from the surface by the precipitating currents from high altitude pair-filled regions, will survive into a more successful model and provide the ion outflow needed in the shock-acceleration model for the synchrotron emission from plerions.

Before completing my discussion of magnetospheric models, I make a brief digression to the Cheng et al. (1986a, b) outer gap model, mainly because this is the only "theory" proposed so far which even crudely explains the whole high frequency photon spectrum of the Crab and Vela pulsars. It also predicts that ordinary pulsars will always be invisible at high frequencies. The basic idea is to assume the presence of "vacuum" electric fields beyond the last closed field line, in the "inaccessible" region between the null surfaces. The closed zone is assumed to be so well filled that it acts as perfect conductor; why this is so is not addressed. Then charged particles dropped into this zone radiate $\gamma$-rays (either by curvature radiation or by inverse Compton emission) which convert into pairs, mainly through the collision of hard $\gamma$-rays with softer photons. Initially, these soft photons are just assumed to be there, but once the cascade is fully developed, the softer synchrotron photons radiated in the cascade itself serve as targets. These pairs themselves accelerate, radiating more photons, with the increase of the photon and pair density occurring in the direction toward increasing radial distance from the pulsar, until finally the pair density becomes sufficient to short out $E_{\|}$at all larger radii. The gap ejects a positron current toward the outside world, and sends an electron current back toward the star, thus offering another suggested scheme for constructing an open circuited model. However, in the working models, only about $10 \%$ of the Goldreich-Julian current passes through the gap, so the current hypothesized in the Arons and Scharlemann model cannot be balanced this way.

The nice feature of these ideas is that they lead to an emergent spectrum approximately like the phase averaged $X$ - and gamma-ray spectra of the Crab and Vela pulsars (if one is generous about order of magnitude discrepancies between theory and observation in the $1-\mathrm{MeV}$ region of the spectrum). As a result, I am intrigued with the thought that outer magnetosphere cascades may be occurring, at least in those pulsars with the smallest $P$ and largest $\dot{P}$. However, I am skeptical of the gap electrodynamics, with the acceleration occurring along the magnetic field. One reason is that in order to get sufficient $\boldsymbol{J} \cdot \boldsymbol{E}$ power in their gap, Cheng et al. are forced to assume the electric field along $B$ in the region of maximum current flow has strength comparable to the maximum vacuum field assumed in their model. Yet the current forms mostly at the outer edge of the gap, where the rapid pair formation suppresses $E_{\|}$to a small fraction of its maximum value. Thus, they overestimate the power in the model's radiative output by at least an order of magnitude, and perhaps much more, depending on the effectiveness of the pairs in shorting out the electric field. In addition, a lot of the model's energy is spent on forming a precipitating pair plasma, whose impact on the surface heats part of the polar cap. When I do the sums on their model of the Vela pulsar, I find they predict too much soft photon flux from the polar caps, by a factor of a few. While the photon emission from outer magnetospheric cascades may have something to do with the observed pulsed hard photon emission from the Crab and Vela pulsars, my own feeling is that some other kind of particle accelerator in the outer magnetosphere is needed.

All of these "outer gap" ideas turn on the observation of Holloway (1973) and of Okamoto (1974), that the region between the null surfaces is inaccessible to particles from the surface. Recently Muslimov and Tsygan (1990) suggested that general relativistic effects on the electrodynamics near 
the surface can be more important than the effect of field line curvature, in the extraction of a relativistic space-charge limited flow from the stellar surface. In particular, they show that a relativistic beam might be extracted on the unfavorably curved as well as the favorably curved field lines-this with $\boldsymbol{E} \cdot \boldsymbol{B}=0$ at the surface but with $\partial E_{\|} / \partial s$ about an order of magnitude bigger than is created by the effect of favorable curvature alone, in pulsars with standard periods ${ }^{5}$. This effect is of no help to populating the inaccessible zone when pair creation is absent (long periods), since the effects of general relativity decline in proportion to $r^{-1}$ while unfavorable curvature becomes more important in proportion to $r^{1 / 2}$, with gravity becoming unimportant above several stellar radii. Thus a chargeseparated plasma cannot propagate relativistically all the way to distances $\sim R_{\mathrm{L}}$. But, with the rapid increase of $E_{\|}$with height, pair creation can intervene, to allow a quasi-neutral plasma which carries a field-aligned current to flow out into the formerly inaccessible region where Arons (1983a) and Cheng et al. (1986a, b) have tried to place quasi-vacuum gaps. If an outflow model of this sort really can be constructed, we would have a return to the theorists preferring round beams, in accord with the most recent drift of phenomenological studies, but we have to look deeper than gaps to find the source of the outer magnetospheric stress which drives the current and the wind.

\section{Closed current models with pairs}

There have been two campaigns of which I am aware which attempted to marry low altitude, pair outflows with outer magnetospheric stresses which can drive the current flow. The first is the work by Beskin, Gurevich and Istomin (1983). These authors solved a model of magnetospheric structure in which they assume a relativistic plasma, made entirely of pairs, comes out along all polar field lines, with no preference for the favorably or unfavorably curved regions. This plasma has density large compared to $\eta_{\mathrm{R}} / e$; therefore, there is no problem with filling the inaccessible region, at least along field lines connected to the source (they do not address filling the inaccessible region where the field lines close well inside the light cylinder). They constructed the magnetospheric fields for general obliquity, not only the aligned rotator, using a special simplifying assumption. In order to have a linear equation for the poloidal magnetic field, they assumed the rotation velocity of the magnetosphere is everywhere the same (equivalent to the voltage drop in a surface gap being independent of distance

\footnotetext{
${ }^{5}$ For pulsars with short periods, such as the Crab, the effects are about comparable.
}

from the magnetic axis), and also assumed the current density passing through such a gap is uniform. Their model is completely determined by the requirement that the magnetic field be continuous at the light cylinder-no appeal is made to the requirement that energy flow to infinity.

The essential result is a structure morphologically similar to the closed current models of Rylov and of Mestel et al.. Plasma streams out relativistically, but the rotation is uniform (and for our purposes, can be considered as co-rotation with the star) until a thin boundary layer is reached at radii comparable to $R_{\mathrm{L}}$. Here, the particle energies are so high that they are no longer tied to the field lines, but are deflected by the inertial drifts into a thin intense current layer, where the plasma energy density is comparable to that of the electromagnetic field. After flowing across the magnetic field, the particles with the same sign as those of the main current extracted from the star return to the star as a current along the boundary with the closed zone.

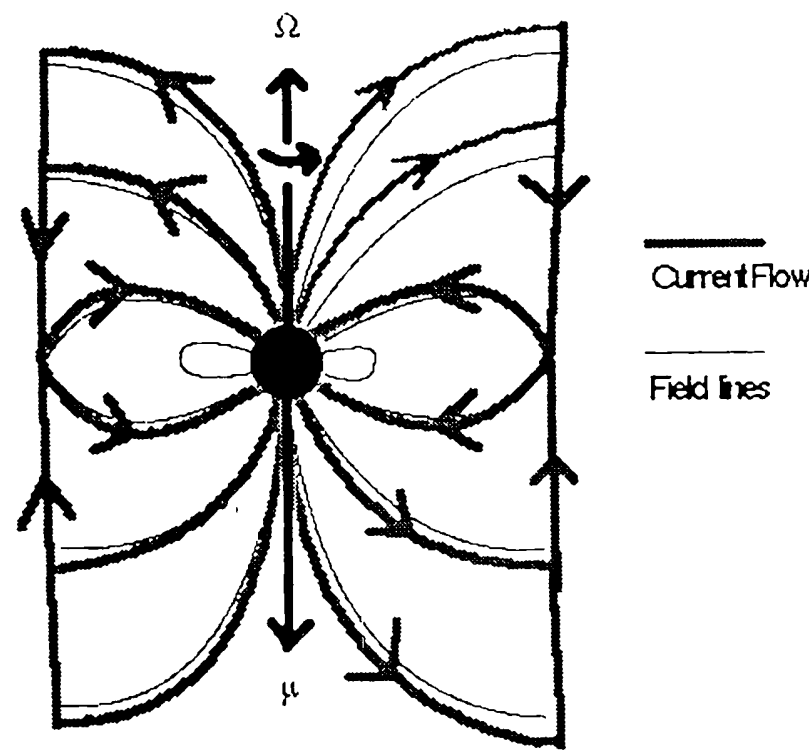

Figure 5 Current flow pattern in the closed model of Beskin, Gurevich and Istomin.

Once again, the effect of a non-corotation acceleration in the outer magnetosphere is essential to establishing a closed current configuration. Because of the high pair density assumed in the outflow from the surface, this acceleration is in a thin boundary layer, rather than in the spatially distributed regions proposed by Rylov and by Mestel et al.. One other important feature established is a required current-voltage characteristic, which constrains the surface gap where the pair plasma is produced:

$$
\Phi=\Phi_{\text {cap }}\left[1-\left(1-\frac{J_{\|}^{2}}{J_{M}^{2}}\right)^{1 / 2}\right]
$$


where $J_{M}$ a characteristic current approximately equal to the Goldreich-Julian current $c \eta_{R}$.

This theory contains a number of new physical ideas. It contains the interesting prediction that the magnetosphere tends to evolve toward having the magnetic moment orthogonal to the rotation axis, with torque declining with increasing obliquity $\chi$, the opposite of what we learned from the vacuum theory. Since their magnetospheres are dense, the vacuum displacement current pattern is irrelevant, and stars close to alignment spin down perfectly well, consistent with Lyne and Manchester's (1988) identification of pulsars with broad beams as being seen almost pole on, yet still having large torques. Indeed, Beskin et al. (1984) have argued that the millisecond pulsars have small $\dot{P}$ not because the field is weak, but because they are almost orthogonal rotators! While entertaining, I doubt this is true, because, as I argued in Chapter I, if counteralignment were working on the spin down time scale, there would be many more orthogonal rotators in the general collection of pulsars than there actually are. A related feature is the ability of the boundary-layer currents to shield off the magnetic field from the outside world and confine it within the magnetosphere. In common with other closed loop models, the star does not lose rotational energy by a large scale Poynting flux. This might turn out to be a good thing, since it provides a third method of understanding how a low $\sigma$ wind could form. Finally, I am intrigued by the properties of the boundary layer as a means of introducing a particle accelerator in the outer magnetosphere without having to introduce an "outer gap". Perhaps the plasma dynamics here will lead to a dynamically successful means of understanding the high energy pulsed emission from pulsars, which will have different predictions from the existing gap models.

There have been suggestions that this theory is "the" solution of the pulsar magnetosphere problem (e.g., Ginzburg, personal communication). My own opinion is that despite the large amount of mathematics expended on the model, it is not a correct theory, as presently developed.

1) These authors have adopted the vacuum gap first suggested by Ruderman and Sutherland (1975) and elaborated by themselves (Gurevich and Istomin 1985b) as the surface source of plasma. Their reason comes from their current-voltage relation, which is imposed by the global magnetospheric structure. This characteristic requires currents $J_{\|} \ll J_{M} \sim c \eta_{R}$, in order to form a surface gap with $\Phi_{\text {gap }} \ll \Phi_{\text {cap }}$, as is required by the pair creation theory. Low current $\left(J_{\|} / J_{M} \sim 0.2\right.$ may be $O K$ for a really active pulsar like Vela, while in fact $J_{\|} / J_{M} \sim 1$ is needed for pulsars approaching cutoff. Their current-voltage characteristic is not consistent with the $J_{\|}=c \eta_{R}+1 \%$ corrections that appears in the relativistic space charge limited flow models. The gap models constructed to match this current-voltage characteristic, which include the effects of positron emission from the surface as it responds to bombardment from above, are clearly inconsistent with the lack of surface binding in the crust-all the models presented have $E \cdot B \neq 0$ on the stellar surface.

2) The boundary layer model for return current formation is inconsistent with the emission of the dense winds needed to power the surrounding nebulae. Their explicit solutions show that in the boundary layer, the radial velocities of all the particles drop to zero - all the particles of the dense plasma are reflected and trapped within the magnetosphere, presumably to precipitate somewhere onto the surface. Beskin et al. never face up to this. They simply say verbally that plasma will leave and energy will be carried out in the form of the flung off pair plasma and "magnetosonic waves", in spite of the lack of any outflow in their own solutions. Because of the lack of outflow in the outer magnetospheric solution, they evaluate the torque on the star not by considering energy flux across an exterior surface, but by looking at the Lorentz forces on the stellar surface contained in their current flow pattern. In a complete theory, these two methods must give the same answer, while in theirs, the energy flux across a surface well outside of $R_{\mathrm{L}}$ is zero, as far as the calculations go. Thus, the results presented actually do not pass the first test of a magnetospheric model, that of producing an energy out-. flow to infinity which can carry away the stellar angular momentum. Possibly their boundary layer solution can be modified and attached to a stellar wind, but just how this could be done is unknown to me. In addition, the theory as presented would give a wind without the heavy ions that I have suggested are necessary to understand the nonthermal excitation of plerions. Here, perhaps a proper treatment of the effects of the precipitating particles would lead to the extraction of ions from the star, along the lines suggested by Arons (1983a).

3) This theory may have trouble with either the limits on $\gamma$-ray emission, or the heating of the surface by precipitation onto the star. The plasma energy density in their boundary layer is comparable to $B^{2} / 4 \pi$, as is necessary if a strong transverse current is to be formed. The energy per particle is $E_{\mathrm{m}} \approx e \Phi_{\text {cap }} / \kappa \cong 1.5 \times 10^{13} \mu_{30} / P^{2} \kappa_{\mathrm{bl}}$, where $\kappa_{\mathrm{bl}}=$ $\left(e n_{ \pm} / \eta_{\mathrm{R}}\right)_{\mathrm{bl}}$ is the multiplicity of pairs which participate in the boundary-layer flow. If the whole pair plasma participates in the boundary layer and little is lost in the wind, $\kappa_{b l} \sim \kappa \sim 10^{3}$. When accelerating across the field, these particles emit curvature radiation. If $\kappa_{\mathrm{bl}}<200 \mu_{30}(0.1 \mathrm{~s} / P)^{7 / 3}$ seconds, 
the boundary layer flow is radiation reaction limited and the whole spin down energy loss of the pulsar would be emitted as curvature photons, whose spectrum would extend up to

$$
\begin{aligned}
\varepsilon_{\max } & \sim 90 \mu_{30}^{3}\left(\frac{0.1 \mathrm{~s}}{P}\right)^{7}\left(\frac{200}{\kappa_{\mathrm{bl}}}\right)^{3} \mathrm{MeV} \\
& \approx 90\left(\frac{0.1 \mathrm{~s}}{P}\right)^{\frac{\mu \nu}{2}}\left(\frac{\dot{P}}{10^{-14}}\right)^{\frac{3}{2}}\left(\frac{200}{\kappa_{\mathrm{bl}}}\right)^{3} \mathrm{MeV}
\end{aligned}
$$

with the photons radiated into most of the sky. This much $\gamma$-ray radiation is forbidden by the upper limits quoted in chapter I, and in the case of the Vela pulsar, is forbidden by the hard X-ray limits from HEAO-A, since about $0.1 \%$ of the spin down energy would be radiated into the HEAO-A band. On the other hand, if $\kappa_{b l}>200 \mu_{30}(0.1 \mathrm{~s} / P)^{7 / 3}$, the boundary layer loses little energy to radiation, but now the return currents precipitating down to the star carry and deposit a luminosity $\left(\kappa_{\mathrm{bl}} / \kappa\right) \dot{E}_{\mathrm{R}}$ into the stellar surface. If the whole plasma participates in the return current flow, the Einstein limits are violated. Clearly, no more than a few percent of the particles can participate in energetic precipitation onto the star, and the existing limits on pulsed X- and gamma-ray emission can be met only if the total pair multiplicity exceeds $10^{4}$. Such high multiplicities may indeed be appropriate for the short period objects where the existing photon limits apply (Arons 1981b, Gurevich and Istomin 1985b). The main point I want to draw here is that the hard photon observations create important constraints on models with closed current flow, including this most sophisticated member of the closed loop morphology.

Finally, I briefly mention interesting calculations by Shibata (1990 and references therein). He attempts to include pair creation in a closed loop model, in which space-charge-limited current leaves the star with nonrelativistic speeds, as in the Rylov and Mestel et al. models, then, at large radius, accelerates to light speed and radiates enough $\gamma$-rays to short out $E_{\|}$. In the outer magnetosphere, he introduces an outer gap, whose non-corotation stress causes the formation of a closed current loop which precipitates back to the star along the boundary of the closed zone. In his most recent work, he has presented an interesting lumped circuit model incorporating these elements, as well as a load representing the wind which must carry away the angular momentum, and attempted to find solutions where the system could operate in a DC manner. Like Beskin et al., he finds a current-voltage characteristic which is inconsistent with relativistic space-chargelimited-flow from the stellar surface, if the system is really steady state in the co-rotating frame. I find this way of looking a things to be very enlightening, and I prefer his model's use of space-chargelimited flow to the appeal to vacuum gaps at the surface, but so far as I can tell from his papers, the acceleration to relativistic velocities and pair formation occurs at altitudes too high to be of use to radio emission models. Furthermore, essentially the same remarks concerning the $\gamma$-ray and surface $\mathrm{X}$ ray emission constraints as I made about the models of Beskin et al. apply to this work too, as do the questions about how to form a wind.

\section{Consequences and the future}

\section{Box scores}

Where does all this leave us? I started with the question of why pulsars do not bark in the night. The various attempts made to satisfy the basic fact of spin down without much photon emission have led to varying success in producing an environment which shows us only a radio whine rather than a loud bark. In table 3 I summarize the successes and failures of the proposed schemes touched on here. An " $X$ " in an entry means the model satisfies present limits or detections, or has desirable features, while a blank means it fails. I should mention I have left out some of the more exotic ideas, such as external disks penetrating the magnetosphere (Michel and Dessler 1981), which seem to me to have no chance of surviving throughout a pulsar's life, if they were ever present in the first place.

\begin{tabular}{|c|c|c|c|c|c|}
\hline & $\begin{array}{l}\text { Energy } \\
\text { lost in } \\
\text { wind } \\
\text { (ions) }\end{array}$ & $\begin{array}{c}\gamma \\
\text { ray } \\
\text { limit }\end{array}$ & $\begin{array}{l}\text { Soft } \\
\text { X-ray } \\
\text { limit }\end{array}$ & $\begin{array}{c}\text { Dense } \\
\text { polar } \\
\text { relativ- } \\
\text { istic } \\
\text { outflow }\end{array}$ & $\begin{array}{c}\mathbf{E} \cdot \mathbf{B} \\
=\mathbf{0} \\
\text { at } \\
\text { star }\end{array}$ \\
\hline Vacuum & & $x$ & $X$ & & \\
\hline $\begin{array}{l}\text { Static } \\
\text { atmo- } \\
\text { sphere }\end{array}$ & & $X$ & $\mathbf{X}$ & & \\
\hline $\begin{array}{c}\text { Closed } \\
\text { loops, } \\
\text { no pairs }\end{array}$ & & & & & $\mathbf{X}$ \\
\hline $\begin{array}{c}\text { Open } \\
\text { currents } \\
\text { with } \\
\text { pairs, } \\
\text { outer } \\
\text { gap }\end{array}$ & 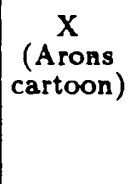 & $\begin{array}{c}\mathrm{X} \\
\text { (Cheng } \\
\text { et al.) }\end{array}$ & & $\underset{\mathbf{X}}{\mathbf{X}}$ & $\begin{array}{l}\mathbf{X} \\
\mathbf{X}\end{array}$ \\
\hline $\begin{array}{c}\text { Closed } \\
\text { currents } \\
\text { with } \\
\text { pairs }\end{array}$ & $?$ & maybe & maybe & $\mathbf{X}$ & $\underset{\text { (Shibata) }}{\mathrm{X}}$ \\
\hline
\end{tabular}

Table 3 


\section{Future work}

Clearly, this subject is not endeu! I he most obvious need is for much more sensitive observations of high frequency photons, which will do better at constraining and probing the various ways in which particle energization must occur in these magnetospheres. As you might suspect, I am particularly excited about the ability of soft X-ray telescopes, and possibly the Hubble Space Telescope working in the ultraviolet, to put constraints on models through studies of polar cap heating. I hope the $\gamma$-ray and hard X-ray experiments on the Soviet-French Granat satellite, on the Gamma Ray Observatory and on Spectrum $\mathrm{X}-\gamma$ and on XTE will improve our knowledge of non-thermal high energy emission from the magnetosphere. Finally, although the observational constraints are more remote, I hope the theories of pulsar radio emission can be developed to make quantitative contact with rádio observations of relevance to the physical state of the plasma. Here, I think study of the rapid fluctuations showing up in pulsar microstructure, and of the circular polarization of the subpulses will be the most revealing, where no radio-emission theory has yet dared to tread in a believable manner.

Making progress on the theory of radio emission is a formidable task, perhaps exemplified by the controversy over the existence of a curvature maser. To illustrate the issue, we know in the theory of curvature emission from a single particle that one can calculate the radiation at high harmonics of the fundamental frequency by solving exactly for the radiation fields of a charged particle moving on a circle, whose radius equals the local radius of curvature of any more general path. Alternatively, one can compute the high harmonic emission directly, by taking advantage of the strong beaming of the photons around the instantaneous velocity vector. These approaches agree, simply because the radiation is strongly beamed. Yet in a dense plasma, calculations of the maser emission due to motion of the whole plasma along a curved field, done by taking direct advantage of the beaming (Beskin, Gurevich, and Istomin 1987) do not agree at all with exact solutions for particles moving in a circular orbit (e.g., Asseo et al. 1983). Modeling the physics of pulsar radio emission will be a task for the future until simple issues like this are resolved.

\section{Better development of closed/open models}

My expectation is that this field will really make progress only when the high energy photon obser- vations start shedding clearer light on the particle acceleration physics in the magnetosphere. In 10 years time, the next of the apparently decennial meetings sponsored by the IAU on pulsar physics might be talking about quite different concepts, in response to what I fondly hope is a tide of useful data from the new generation of satellites, instead of the tantalizing tidbits we have now. However, for what it is worth, I will set out my shopping list of theoretical tasks.

In the first place, does the ion-doped wind model really explain plerions? The evidence I have presented here is certainly headed in that direction, but we need to do much better, both in the theory and simulation of the acceleration process and in its application to models of the plerions, before we can really use this kind of information as a detailed constraint on pulsar magnetospheres.

Further investigation of the possible currentflow layers near the light cylinder, and their relation to wind formation, is clearly needed, in the context of the pair-creation models. The suggestions coming from the brave souls who have pursued the closed current-flow models have many intriguing aspects, but they especially need development in directions which show how the energy can be lost in the form needed to excite the surrounding medium.

Finally, within the context of understanding the closed loop models, some means must be found to reconcile the current-voltage characteristic imposed by the magnetosphere with relativistic spacecharge-limited flow at the surface, so as to allow the generation of the dense outflowing plasma suggested by the radio radiation. The most likely solution to my way of thinking is to give up strict time steadiness in the corotating frame (Arons 1979), so that the pulsar could send out plasma with an inappropriate current, then shut off when the magnetosphere sends back its signal, and repeat the cycle. The fact that subpulses are unsteady on time scales corresponding to the Alfvén wave transit time between the star and light cylinder can be usefully interpreted in terms of such an unsteady magnetosphere (Arons 1981c, Arons 1981b, Rylov 1982).

\footnotetext{
Acknowledgments: My efforts in preparing this review and on studies of relativistic shock waves were supported in part by NSF grant AST-8615816 and by IGPP-LLNL grant 90-14, both to the University of California at Berkeley. The work on shocks was also supported by NASA astrophysical theory grant NAGW1301. Part of the work was performed under the auspices of the U. S. Department of Energy at the Lawrence Livermore National Laboratory under contract W-7405-Eng-48.
} 